\title{
Level 6: Services und Aktivitåten mit Games
}

Kommen wir nun zum nächsten Level: Aktivitäten und Projekte im Bereich Gaming. Bevor ich Ihnen einige Vorschläge für neue Gaming-Services unterbreite, möchte ich zuerst einen Blick auf das bisher Gelesene werfen. Sie haben in einem ersten Schritt gelernt, was mit dem Begriff Gaming gemeint ist. Sie haben zudem einen Einblick in die Hardware und daran anschließend in die Software bekommen. Schließlich habe ich die Möglichkeiten, aber auch die Grenzen einer klassischen Bestandsarbeit im Bereich Gaming aufgezeigt. Gerade der letzte Punkt ist für diesen Abschnitt relevant.

Ich hoffe, ich habe Ihnen aufzeigen können, dass die Fokussierung auf den Bestand im Bereich Gaming wenig bringt. Natürlich werden Sie auch weiterhin einen Bestand haben. Aber dieser wird zunehmend an Bedeutung verlieren bzw. in Teilen nicht mehr realisierbar sein. Der digitale Vertrieb wird auch weiterhin dafür sorgen, dass sehr viele Inhalte im Bereich Gaming nicht auf einem physischen Datenträger zur Verfügung stehen. Zudem sind die Spiele zunehmend an die digitalen Vertriebsplattformen bzw. die Accounts der Spieler gebunden. Ein Verleihen, als digitales Medium vergleichbar mit den Verleihangeboten für eBooks, wird also ebenfalls nicht realisierbar sein. Schließlich gibt es neue Bezahlmodelle für Computerspiele wie z. B. Freemium und Free-to-Play, bei denen man das Spiel kostenlos spielen kann und nur für besondere Fähigkeiten, Ausrüstungsgegenstände etc. im Spiel bezahlen muss. Spätestens dann, wenn Spiele kostenlos verfügbar sind bzw. wenn das Geschäftsmodell der Games-Industrie weg vom Verkauf des Spiels und hin zum Ermöglichen des Zugangs zum Spiel oder einzelner Elemente davon umgestellt wird, ist das Verleihen von Games endgültig ein Nischenthema. Für Bibliotheken bedeutet dies, dass sie sich zum einen überlegen müssen, was sie außer der klassischen Bestandsarbeit im Bereich Gaming tun möchten. Zum anderen müssen sie sich eine Strategie für die Übergangsphase überlegen. In dieser Übergangsphase geht es darum, eine zukunftsweisende Bestandsarbeit und die Entwicklung völlig neuer Services miteinander zu verbinden. Ich betone das Thema Bestand deshalb so deutlich, weil ich in meinen Workshops sehr oft erlebe, dass Bibliotheken, die einen eigenen Bestand im Bereich Gaming haben, der Meinung sind, dass sie damit das Thema Gaming abgeschlossen haben. Aber dem ist nicht so. Im Gegenteil: die Bestandsarbeit kann nur ein erster Schritt sein.

Gaming in Bibliotheken sollte irgendwann die gleiche Relevanz haben wie Bücher in Bibliotheken. Und auch wenn Sie es sich vielleicht im Moment nicht vorstellen können, so gibt es doch weitaus mehr Möglichkeiten als Sie glauben mögen.

Allerdings benötigt dies ein Umdenken von Seiten der Bibliothek. Es bedeutet, dass man als Bibliothek nicht mehr den Bestand als zentral wichtiges Thema versteht. Das heißt auch, dass man sich weitaus mehr Know-how aneignen muss und dies in einem Bereich, in dem Bibliotheken trotz aller Erfolge immer noch am Anfang stehen.

Das Arbeiten mit dem Thema Gaming ist also eine langfristige, folgenreiche und vor allem strategische Entscheidung einer Bibliothek, denn es wird auch bedeuten, dass man sich von anderen Angeboten trennen wird. Gaming ist keine freiwillige $\mathrm{Zu}$ satzaufgabe. Es ist eine gleichwertige Aufgabe bzw. ein gleichwertiges Thema zu allen anderen Themen in der Bibliothek.

Die gute Nachricht ist die, dass viele der Services und Aktivitäten, die ich Ihnen vorschlagen möchte, bereits vorhandenen Services von Bibliotheken ähneln. Genauer gesagt werden in manchen Fällen bereits vorhandene Services um das Thema Gaming erweitert.

Natürlich werden nicht alle Ideen zu Ihnen passen. Jede Bibliothek muss letztlich eine individuelle Auswahl treffen. Deshalb habe ich die Vorschläge auch nicht unter- 
teilt. Die Möglichkeit, sich als Bibliothek im Bereich Gaming erfolgreich zu positionieren, hat nichts mit der Größe und nichts mit der Ausrichtung zu tun. Einzig relevant ist die Frage, ob Sie dieses Thema bearbeiten möchten oder nicht.

\section{Einfache Gaming-Events}

Beginnen wir die Präsentation der Beispiele mit einfachen Gaming-Events. Damit sind Veranstaltungen gemeint, die mit einem geringen Aufwand einmalig in der Bibliothek stattfinden. Es geht dabei um zwei Punkte:

1. Die Bibliothek kann einen Gaming-Event ausprobieren. Es geht also um das Sammeln von Erfahrungen. Gerade wenn Sie mit dem Thema beginnen, sollten Sie sich nicht übernehmen. Sie müssen nicht gleich das unglaublich großen und erfolgreichen Event durchführen. Aber es ist wichtig zu verstehen, was da auf Sie zukommt.

2. Sie können ausprobieren, ob es in Ihrem Umfeld überhaupt Interesse am Thema gibt. Wenn Sie am Anfang kein Interesse an dem Thema erfahren, heißt das nicht automatisch, dass man es nicht will. Es kann auch gut sein, dass Sie mit Ihrer Kommunikationsstrategie die neuen Zielgruppen gar nicht erreichen. Auch um dies herauszufinden, eignet sich ein kleinesr Event sehr.

Ein kleinesr Event sollte also einen Prototyp-Charakter haben. Da es um Gaming geht, sollte es bei dem Event ebenfalls um das Spielen gehen. Nehmen Sie am besten eine Gaming-Konsole wie die XBOX 360 und die Playstation 3 und laden Sie Ihre Kunden zum Spielen ein. Der Event muss gar nicht lange dauern. Es reicht vollkommen, wenn Sie 2-3 Stunden einplanen. Je nachdem können Sie den Event während oder außerhalb der normalen Öffnungszeiten durchführen. Ich habe sehr oft erlebt, dass sich so ein kleinesr Event während der Öffnungszeiten schon deshalb lohnt, weil dann weitaus mehr Menschen etwas davon mitkriegen. Selbst wenn diese Besucher nicht selbst spielen, können diese Menschen immer noch als Zuschauer dienen.

Denken Sie immer daran, dass es nicht nur die Gamer, sondern auch die Zuschauer gibt. Ein Gaming-Event ist nicht nur ein Event, sondern ebenso ein Zeichen. Sie zeigen damit, dass Sie sich mit dem Thema beschäftigen möchten. Es kann auch sehr gut sein, dass Sie auf diesem Weg Menschen kennenlernen, die ihrerseits im Bereich Gaming aktiv sind. Mit diesen Menschen könnten Sie sich vernetzen und in Zukunft kooperieren.

Im Rahmen dieses Events sollte es nur darum gehen, dass jeder Besucher ein bisschen spielen kann. Es geht also nicht um einen Wettbewerb oder gar einen groBen Gaming-Day. Es geht um ein Kennenlernen, um einen Austausch und zugleich um die Möglichkeit, etwas Neues auszuprobieren.

Ich würde Ihnen empfehlen, Spiele auszuwählen, die folgenden Kriterien entsprechen:

1. Die Spiele sind leicht zu spielen. Jeder kann ohne Training mitmachen.

2. Die einzelnen Runden sind kurz - maximal fünf Minuten. Auf diesem Weg können viele Menschen das Spiel ausprobieren.

3. Das Spiel wird über eine Bewegungssteuerung also entweder Wiimote, XBOX 360 Kinect oder Playstation Move gesteuert. Damit ist es zum einen einfacher, das Spiel zu spielen (die meisten Spiele für die Steuerungen sind einfach zu spielen), und zum anderen entsteht ein höherer Zuschauer-Faktor. Für die anderen Be- 
sucher der Bibliothek ist es viel spannender, Menschen zu sehen, die sich bewegen als solche, die auf einem Stuhl sitzen und Knöpfe an einem Controller drücken. Die Interaktion wird also zum Teil des Zuschauens.

4. Die Spiele erzählen keine komplexe Geschichte. Ansonsten verstehen die Spieler zwar die Aufgaben, aber nicht die Geschichte.

Es ist wichtig, dass ein Großteil Ihres Teams die Spiele vor dem Event ausprobiert bzw. selbst spielt, damit man die Teilnehmer unterstützen und zugleich aktivieren kann. Wenn möglich können Sie auch einen oder zwei Kollegen am Tag des Events mit der Unterstützung und der Aktivierung der Teilnehmer betrauen. In vielen Fällen sind es Kinder und Jugendliche, die die Spiele ausprobieren. Natürlich ist dies ein Erfolg, aber es wäre noch besser, wenn Sie auch Erwachsene aktivieren könnten. Der größte Erfolg wäre, dass Kinder und Jugendliche zusammen mit Erwachsenen spielen. In meinen Events habe ich immer feststellen können, dass man gerade junge Gamer sehr einfach für neue Spiele begeistern kann. Es ist dabei nur wichtig, dass Sie als Bibliotheksmitarbeiter in der Sprache der Kinder und Jugendlichen sprechen. Gerade junge Gamer fokussieren sich zuerst gerne auf die Spiele, die sie bereits gespielt haben. Sie fühlen sich in diesen Games sicher und gehen von einer größeren Chance aus, das Spiel auch gewinnen zu können. Ein Spiel, welches ich in solchen Situationen immer gerne nutze, ist „Raving Rabbits - alive and kicking“. Dieses Spiel wird auf der XBOX360 mit dem Kinect-System gespielt. Sie können dabei eine größere Gruppe an einer Konsole spielen lassen. Das Spiel hat einen einzigartigen Humor - der sowohl Kids als auch Erwachsene begeistert. Im Laufe des Spiels treten immer zwei Spieler zu kleinen bewegungsintensiven Games an. Sowohl das Spielen als auch das Zusehen macht großen Spaß. Die Verlierer müssen zur Strafe bestimmte Aufgaben erfüllen wie z. B. das laute und öffentliche Gackern wie ein Huhn. Dieses Spiel ist gerade bei jüngeren Gamern nicht so bekannt wie z. B. „Need for Speed“ oder „Fifa“. Es bedarf also einer Person, die die Spieler aktiviert, das Spiel auszuprobieren. Hat man dies aber geschafft, dann sind die Spieler in den meisten Fällen mehr als begeistert.

Wenn Sie den Event durchführen, sollten Sie sich vorher überlegen, was Sie auf die Frage antworten möchten, ob es weitere Aktivitäten im Bereich Gaming geben wird. Jede Frage dieser Art gibt Ihnen die Möglichkeit, mehr Menschen darüber zu informieren, dass Sie ein Gaming-Ort werden, natürlich nur, wenn Sie dies auch werden wollen. Allerdings gehe ich davon aus, dass Sie einen Gaming-Event erst dann veranstalten, wenn Sie sich langfristig mit dem Thema Gaming beschäftigen. Wie gesagt, der größte Fehler, den Sie machen können, ist der, den Besuchern zu sagen, dass Sie nicht wissen, ob und wenn ja wie es im Bereich Gaming weitergeht. Des Weiteren können Sie in diesem Moment versuchen, die fragenden Besucher zu aktivieren, Ihnen bei der Umsetzung der Gaming-Aktivitäten zu helfen.

Natürlich kann es auch passieren, dass Sie für diese Aktivitäten kritisiert werden. Sie müssen immer daran denken, dass es Menschen gibt, die ein Bild von einer Bibliothek haben, welches die Bibliothek als Ort der Ruhe und der Bücher sieht. Viele dieser Menschen sind bereit, diese „ihre“ Bibliothek zu verteidigen. Versuchen Sie, diesen Menschen klarzumachen, dass die Bibliothek nicht zu einer Spielhalle wird, dass aber Gaming ein ebenso bedeutendes Thema ist wie Bücher oder irgendetwas anderes.

Es reicht völlig aus, während dieses Events nur eine Konsole aufzustellen und die Besucher spielen zu lassen. Sie müssen dabei nichts Weiteres tun. Wichtig ist, dass Sie einen Ort für das Thema Gaming schaffen und zusehen und zuhören. Und dass Sie versuchen, Ihr Team und Ihre Kunden für das Thema zu begeistern. 


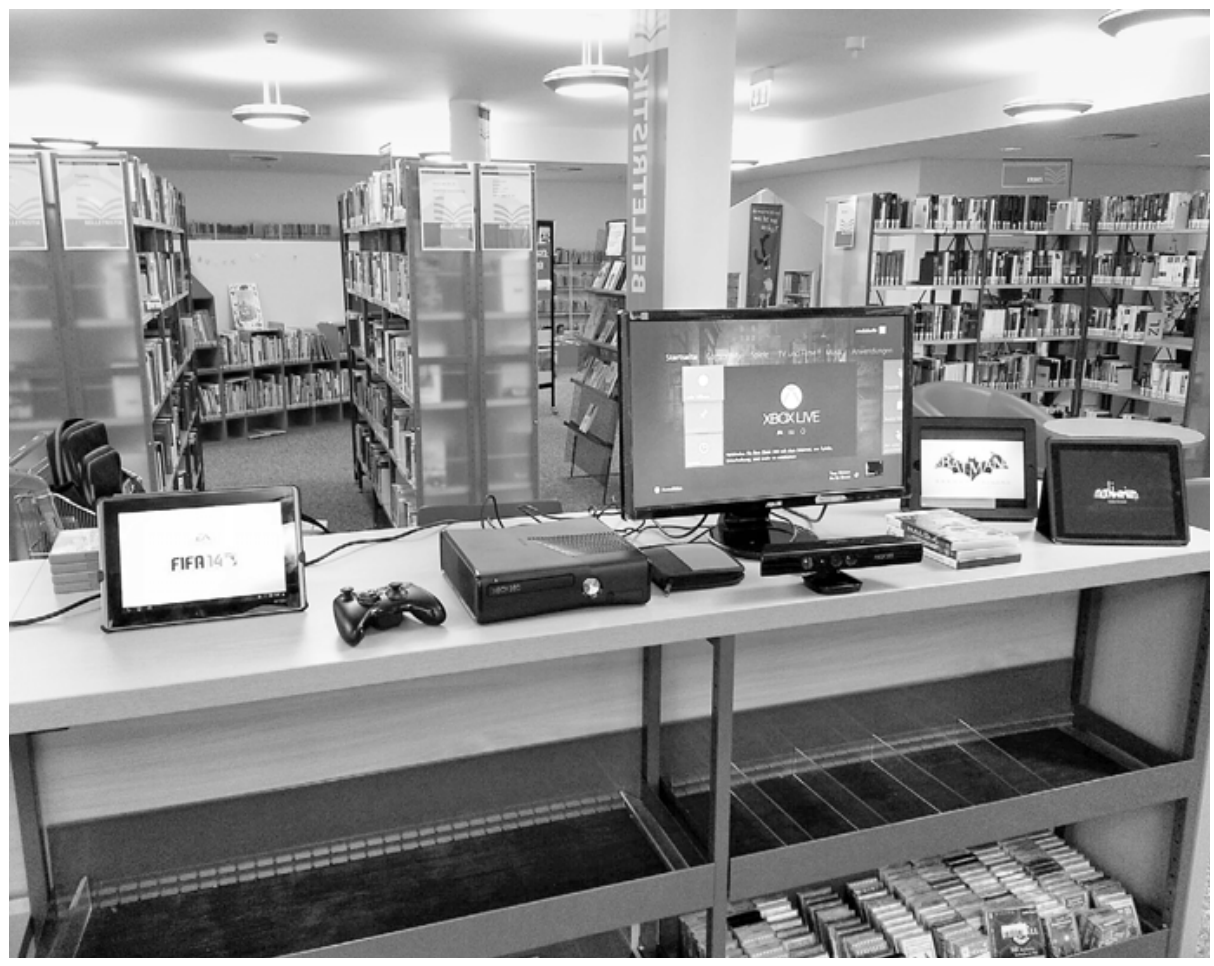

Abb. 25 Gaming-Station bei einfachem Gaming-Event

\section{Zusammenfassung:}

Name der Veranstaltung: Einstiegs-Event Gaming

Zielsetzung: Prototyp Gaming-Events, Aktivieren der Kunden und Mitarbeiter, Ausprobieren und erste Erfahrungen sammeln

Benötigte Ressourcen: 1x Konsole inkl. Monitor, Controller, Verkabelung, Lautsprecher, Strom, Spiele, Raum

Zeitrahmen: 2-3 Stunden (Vorbereitung ca. 8 Stunden)

\section{Gaming Wettbewerbe}

Wenn wir uns mit Gaming-Aktivitäten beschäftigen, dann kommen wir an GamingWettbewerben nicht vorbei. Der Wettbewerb ist ein elementarer Bestandteil des Gamings. Egal, ob man gegen den Computer, die Zeit oder einen realen anderen Spieler antritt, Gaming ist gleichbedeutend mit Wettbewerb.

Aber Wettbewerb ist nicht nur Teil des Spielens. Wettbewerb hat das Gaming massiv weiterentwickelt. Unter dem Begriff eSports finden regelmäßig professionelle Gaming-Wettbewerbe statt. In verschiedenen Disziplinen bzw. Genres treten einzelne Spieler oder Gruppen gegeneinander an. Einige Spieler können von diesen Wettbewerben sehr gut leben. Sie verdienen im Jahr über $100.000 €$ und sind nicht nur in der Gaming-Szene Superstars. In Südkorea sind die sogenannten Pro-Gamer so bekannt und beliebt wie Pop-Stars und eSports ist zu einem Volkssport geworden. Auch in Deutschland werden solche Wettbewerbe immer beliebter.

Wettbewerbe sind mehr als nur Gaming-Events. Nicht mehr das Spiel, sondern der stattfindende Wettkampf bzw. die Kontrahenten stehen im Mittelpunkt des Inte- 
resses. Gaming-Wettbewerbe sind immer große Veranstaltungen und mancher Besucher kommt wegen den Gamern, andere wiederum möchten einfach die Atmosphäre genießen bzw. die Spieler anfeuern. Gaming-Wettbewerbe ziehen also nicht nur Gamer, sondern auch Wettbewerb-Begeisterte an.

Bevor Sie jetzt nervös werden, möchte ich Sie beruhigen. Sie müssen keinen Event für 5.000 Gäste konzipieren. Sie müssen auch keine Angst haben, dass Sie ein Preisgeld in Höhe von $30.000 €$ für den Sieger akquirieren müssen. Diese von mir beschriebenen Großveranstaltungen sind etwas für große Unternehmen, die sich um nichts anderes kümmern und damit ihr Geld verdienen. Allerdings können Sie einiges von diesen Events lernen.

Wie bereits beschrieben, sind Gaming-Wettbewerbe etwas Besonderes. Sie haben es bei solchen Veranstaltungen mit mehreren Teilnehmergruppen zu tun. Diese sind vergleichbar mit den Zielgruppen bei den schon beschriebenen EinsteigerEvents. Sie haben zum einen die eigentlichen Wettbewerbsteilnehmer, also die Personen, die die Spiele spielen bzw. gegeneinander antreten. Zum anderen sind es die Angehörigen und Freunde der Teilnehmer. Diese werden mit Sicherheit mitfiebern und Sie brauchen dafür den notwendigen Raum. Dann gibt es als dritte Zielgruppe noch die Interessierten. Sie haben keine Verbindung zu den Spielern, sind aber an dem Thema Gaming bzw. Wettbewerben interessiert. Schließlich gibt es noch die normalen Bibliothekskunden, die dem Event positiv, neutral oder negativ gegenüber stehen.

Sie müssen für alle genannten Zielgruppen ein eigenes Angebot entwickeln. Besonders wichtig ist es, Spieler zu finden, die gegeneinander antreten möchten. Die Tatsache, dass Sie einen solchen Event anbieten, bedeutet nicht dass auch automatisch genügend Teilnehmer kommen. Grundsätzlich ist es so, dass die Spieler die Spiele auch ohne Sie spielen können. In den meisten Haushalten stehen heute Konsolen und/oder Gaming-Computer. Die Bibliothek wird also nicht benötigt, um einen Zugang zum Thema Gaming zu ermöglichen. Es stellt sich also die Frage, was Sie den Spielern anbieten können, was sie zuhause nicht bekommen. Hier gibt es verschiedene Ansätze. Zum einen können Sie einen Event anbieten, bei dem es Zuschauer gibt. Des Weiteren können Sie einen öffentlichen Event anbieten. Sie können auch einen Preis und vor allem einen Titel anbieten. Das heißt, das Spielen an sich ist kein Alleinstellungsmerkmal. Sie müssen es zu etwas Besonderem machen.

Zuerst sollten Sie dem Event einen Namen geben. Nennen Sie die Veranstaltung auf keinen Fall „Spielenachmittag“ oder „Jugendspiel“. Es geht um einen GamingEvent. Und es geht darum, einen Sieger zu finden. Ich weiß, dass sich Bibliotheken sehr oft schwer tun, eine etwas „kräftigere“ Sprache zu nutzen.

Es ist also kein Spiel-Wettbewerb, sondern der „ultimative Gaming-Event“. Es geht darum, den großen „Gaming-Champion“ Ihrer Bibliothek zu finden. Zudem brauchen Sie einen offiziellen Preis. Am besten, Sie haben einen kleinen Pokal und natürlich Urkunden. Sie brauchen eine Siegerehrung und einen Spielplan. Sorgen Sie dafür, dass der Ort des Wettbewerbs etwas Besonderes ist. Wenn möglich sollten die Spiele auf einer kleinen Bühne stattfinden. Vielleicht haben Sie ja die Möglichkeit, einen guten Beamer und eine Leinwand zu bekommen, auf die Sie die Spiele projizieren können. Wenn es die Räumlichkeiten zulassen, können Sie einen Raum als „Backstage-Bereich“ nutzen. Hier können sich die Spieler ausruhen und natürlich ein paar Snacks und Getränke zu sich nehmen.

Arbeiten Sie also genau aus, was Ihren Event spannender macht als einen Gaming-Abend zu Hause. Und dann sollten Sie all dies kommunizieren. Das macht Ihren Event zu etwas Besonderem.

Sie haben bezüglich der Struktur des Wettbewerbs zwei Möglichkeiten: 
1. Sie veranstalten einen Event an einem Tag. Dies könnte so aussehen, dass Sie die Spieler in Gruppen einteilen. In diesen Gruppen treten die Spieler gegeneinander an. Die ersten und zweiten Sieger einer jeden Gruppe kommen in die nächste Runde. Dort treten sie wieder gegeneinander an. Der jeweilige Sieger kommt eine Runde weiter bis am Schluss nur noch zwei Spieler übrig sind, die dann im Finale gegeneinander antreten.

2. Sie führen vorab eine Reihe von kleineren Wettbewerben nach einem vergleichbaren Muster durch. Schließlich gibt es ein großes Finale, an dem vielleicht zehn Finalisten teilnehmen, die alle gegeneinander antreten und für jeden Sieg Punkte bekommen.

Auch hier müssen Sie darauf achten, dass die Spiele passen. Es kann aber gut sein, dass einige der Spieler die Spiele des Wettbewerbs schon öfter gespielt haben als andere. Um hier einen Ausgleich zu schaffen, empfehle ich zum einen, die Spiele nicht vorab zu kommunizieren. Zum anderen können Sie auch jeweils zwei Spiele spielen lassen, wobei Sie als zweites Spiel ein sehr exotisches mit äußerst geringem Bekanntheitsgrad wählen. Ziel dieser Herangehensweise ist es, auch unerfahrenen Spielern eine Chance zu geben. Ganz egal welchen Weg Sie wählen, achten Sie darauf, dass Sie den Teilnehmern die Möglichkeit geben, die Spiele auszuprobieren bzw. zu trainieren.

Sie können natürlich auch Teams zusammenstellen. Diese Teams gewinnen gemeinsam Punkte und gewinnen dann gemeinsam den Wettbewerb. Die Zusammenstellung der Teams ist Ihnen überlassen. Wenn Sie Ihren Event nicht nur für Jugendliche und Kinder konzipieren, sondern alle Altersgruppen ansprechen möchten, dann können Sie durch die Teamzusammenstellung bereits einiges erreichen. Sie können z. B. Anreize schaffen, indem das Team Sonderpunkte bekommt, bei dem der Altersunterschied zwischen den Teilnehmern besonders groß ist. Wenn Sie z.B. Teams aus zwei Spielern bilden und ein 14-jähriges Mädchen bringt seinen 65 Jahre alten Großvater mit, dann hätte dieses Team 51 Sonderpunkte gesammelt.

Wenn Sie nur geringe Erfahrung in der Entwicklung und Realisierung von Gaming-Veranstaltungen haben, sollten Sie in einem ersten Schritt eine eintägige Veranstaltung planen. Bei so einer Veranstaltung wird es schwer werden, den normalen Geschäftsbetrieb aufrecht zu erhalten. Ein Gaming-Wettbewerb ist viel zu wild und vor allem zu laut. Andere Bibliothekskunden würden sich mit Sicherheit gestört fühlen. Es ist also eine Veranstaltung, bei der die Bibliothek geschlossen bleibt, es sei denn Sie haben genügend Räumlichkeiten, um beide Bereiche voneinander zu trennen.

\section{Zusammenfassung:}

Name der Veranstaltung: Gaming-Wettbewerb

Zielsetzung: neues und spannendes Veranstaltungsformat, Wettbewerb, Einbindung verschiedener Zielgruppen bzw. Altersklassen

Benötigte Ressourcen: mindestens 4 Konsolen inkl. Monitor, Controller, Verkabelung, Lautsprecher, Strom, Spiele, Raum - Für das Finale: zudem einen Beamer und eine Leinwand

Zeitrahmen: 6-8 Stunden (Vorbereitung ca. 5 Tage) 


\section{Summer Games}

Summer Games sind eines meiner liebsten Konzepte. Dabei sind die Summer-Games eigentlich kein einzelner Event. Es ist vielmehr eine Zusammenstellung von Events und Aktivitäten, bei denen auch bereits vorhandene Projekte der Bibliothek in die Summer Games integriert werden.

Die Idee für die Summer Games kam mir während eines Workshops für Bibliotheken, die sich intensiver mit Gaming beschäftigen wollten. Später habe ich durch Gespräche mit Eli Neiburger von der Ann Arbor District Library weitere Ideen entwickelt und einige der Ideen von Eli Neiburger übernommen.

Die Grundidee der Summer Games ist die, dass man den Sommer, genauer die Sommerferien, dazu nutzen könnte, eine Reihe unterschiedlicher Aktivitäten zu einem Gesamtprogramm den sogenannten Summer Games zusammenführt. Viele Bibliotheken haben bereits ein spezielles Programm für die Sommerferien. Ein Programmpunkt ist sehr oft der „Sommer-Leseclub“. Diejenigen von Ihnen, die in Bibliotheken arbeiten, kennen das Konzept sicherlich sehr gut.

Der Sommerleseclub ist vor allem auf das Buch als Medium fokussiert. Ich möchte das nicht kritisieren aber ich glaube, der Club eignet sich ideal, um ihn durch Gaming zu erweitern. Hierfür gibt es zwei Ansätze:

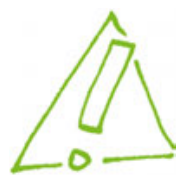

1. Gaming als Inhalt bzw. als Medium: In diesem Fall geht es nicht mehr alleine um das Lesen von Büchern, sondern ebenso um das Spielen von Computerspielen. Es gibt eine Vielzahl an Spielen, die einen sehr großen Textanteil haben. Dabei geht es sowohl um Texte, die die Aufgaben des Gamers beschreiben, als auch um Texte, die die Geschichte des Spiels erklären. In beiden Fällen geht es um Lesen, wobei die Motivation nicht nur das von der Bibliothek verliehene Zertifikat, sondern auch das Spiel an sich ist. Zudem ist die Welt des Gamings voll von Schreiben und Lesen. Und alle diese Bereiche könnten in die Summer Games integriert werden. So können Sie sich von den Teilnehmern die Geschichten aus ausgesuchten Games erzählen lassen. Sie können dies bei älteren Teilnehmern (ab 10 Jahre) auch über einen Blog durchführen. Sie schulen dann auch noch die in der $\mathrm{Zu}$ kunft so wichtige digitale Medienkompetenz. So oder so werden sie Teilnehmer mit hoher Motivation erleben. Zudem werden die Spiele in vielen Fällen näher an der Lebensrealität der Teilnehmer sein als es vielleicht Bücher sind.

2. Gaming oder Gamification als Struktur bzw. Modell für die Summer Games. Wie ich bereits in vorherigen Kapiteln beschrieben habe, gibt es die Möglichkeit, bestimmte Aktivitäten und Programme durch Gaming-Elemente anzureichern. In gewisser Weise hat der Sommer-Lese-Club bereits Gamification-Elemente übernommen. Das Zertifikat ist das Ziel, man hat bestimmte Aufgaben zu erfüllen und bekommt sogar ein Feedback. Jedoch gibt es keinen Wettbewerb, keine übergeordnete Geschichte und keinen Grund, nach den zu lesenden Büchern mit dem Lesen weiterzumachen. Die Summer Games wären also auch ein Versuch, die Lesedauer zu verlängern.

Egal, ob Sie sich für den ersten oder den zweiten oder beide Ansätze entscheiden, ein Summer-Gaming-Club bedeutet, dass es dann nicht mehr alleine um Bücher, sondern auch um das Thema Gaming gehen würde. Es bedeutet auf jeden Fall einen Mehraufwand, da Sie sich auf eine völlig andere Kultur einlassen würden.

Zuerst müssen Sie sich überlegen, was die Teilnehmer tun sollen. Sollen sie Bücher lesen, Games spielen, Geschichten aufschreiben etc.? So oder so müssen Sie genau definieren, was man mit welchen Medien tun kann. Je größer die Möglichkeiten 
für den Teilnehmer sind, desto mehr Menschen werden mitmachen. Sie müssen die Spiele nicht alle im Bestand haben. Sie können ebenso auf Onlinespiele zurückgreifen. Letztlich können Sie jedes Projekt, welches ich in diesem Buch vorstelle, zu einem Teil der Summer Games machen. Jedoch haben wir es beim Sommer-Lese-Club mit dem Thema Leseförderung zu tun. Also sollten Sie ein Konzept erstellen, welches diesen Bereich beleuchtet.

Es geht bei den Summer Games nicht mehr darum, zu beweisen, dass man ein Buch gelesen hat. Alle Medien sind Teil einer Geschichte. Die Teilnehmer sollen bestimmte Aufgaben lösen. Dies kann z. B. das Suchen bestimmter Informationen sein, für die man ein Buch lesen oder ein Spiel spielen muss. Der Beweis für das Lesen bzw. Bearbeiten der Aufgabe liegt dann darin, dass man die richtige Lösung hat. Sie können damit einzelne Elemente eines Buches oder eines Spiels nutzen. Sie können aber auch weitere Aufgaben vergeben. So können Sie kleine Geschenke im OPAC verstecken, die man dann suchen muss. Sie können auch Aufgaben in der realen Welt verteilen.

Nachdem Sie also definiert haben, welche Medien konsumiert werden sollen, müssen Sie überlegen, welche Elemente bzw. welche Inhalte gefunden werden bzw. was die Teilnehmer mit den Medien anfangen sollen. Im Anschluss daran müssen Sie einen „roten Faden“ für die einzelnen Aufgaben entwickeln. Dieser rote Faden verbindet die einzelnen Aufgaben miteinander. Die Alternative ist, dass die Verbindung der einzelnen Aufgaben nur über Punkte geschieht. Der Vorteil dieser Variante ist der, dass Sie eine weitaus größere Auswahl an Aufgaben haben, da Sie diese nicht in eine Geschichte zusammenfassen müssen.

Achten Sie bei der Zusammenstellung der Aufgaben darauf, dass man die Aufgaben auch erfüllen kann. Man sollte die Aufgabe zudem einfach erklären können.

In beiden Fällen entsteht ein Wettbewerb, bei dem am besten verschiedene Fähigkeiten benötigt werden. Es sollte also nicht ausreichen, nur ein Buch zu lesen oder nur ein Spiel zu spielen. Jeder Teilnehmer hat Aufgaben und muss diese durch Lesen von Büchern, Spielen von Spielen, Onlinerecherche, Katalog-Recherche etc. umsetzen. Für das Umsetzen jeder Aufgabe bekommt man Punkte oder kann bestimmte Badges freischalten. Ob das Erreichen der Punkte oder der Wettbewerb im Vordergrund stehen, müssen Sie entscheiden.

Natürlich können Sie das Ganze noch steigern, indem Sie bestimmte Aufgaben verteilen, bei denen man mit anderen kooperieren muss. Diese Kooperationen können Sie dann mit besonders hohen Punktzahlen belohnen.

Die Summer Games ermöglichen es, Leseförderung und weitere Ziele der Bibliotheksarbeit über verschiedene Medien zu erreichen. Neben der Leseförderung können auch Medien- und in Teilen Informationskompetenz vermittelt werden. Diese Herangehensweise ist sicherlich nicht für ganz kleine Bibliotheken alleine umsetzbar. In so einem Fall könnte man Kooperationen zwischen vielen kleinen Bibliotheken erstellen. Es wäre auch möglich, dass die Bibliotheken Teams zusammenstellen, die gegeneinander antreten. In diesem Fall würde jede Bibliothek Aufgaben für alle Teams zusammenstellen.

Wenn Sie so etwas wie die Summer Games durchführen möchten, sollten Sie sich vorher auf jeden Fall ein bisschen mit Game-Design bzw. Game-Mechaniken beschäftigen. Erst dann werden Sie in der Lage sein, ein umfassendes Erlebnis für Ihre Kunden zu entwickeln.

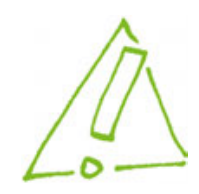




\section{Zusammenfassung:}

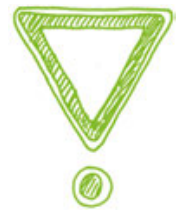

Name der Veranstaltung: Summer Games

Zielsetzung: über die Nutzung von Games und Gamification Leseförderung und Medienkompetenz vermitteln

Benötigte Ressourcen: Computer, Zeit, Ideen

Zeitrahmen: 2-6 Wochen (Vorbereitung ca. 18 Tage)

\section{Online-Gaming}

Den Begriff Online-Ggaming habe ich schon bei der Übersicht über die Genres beschrieben. Deshalb möchte ich ihn hier nur noch einmal kurz zusammenfassen. Online-Gaming meint das Spielen online. Dabei gibt es verschiedene Varianten:

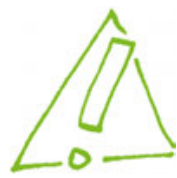

1. Man spielt einen Teil eines Spieles online. Dies ist z. B. bei Multiplayer-Gaming der Fall.

2. Man spielt ein Spiel im Internetbrowser. Dies sind die sogenannten BrowserGames und die sogenannten Social-Games.

3. Man lädt einen Client herunter und dieser fungiert als Schnittstelle zum Spiel, welches auf einem externen Server läuft.

4. Man spielt ein Retro-Game, welches auf einem externen Server läuft. Hier ist der Computer nur eine Schnittstelle zu einem Video-Stream.

Für Bibliotheken bedeutet Online-Gaming eine große Herausforderung. Zum einen wird durch Online-Gaming bzw. den Erfolg dieses Bereiches jede klassische Bestandsarbeit in Frage gestellt. Es gibt schlichtweg keine Möglichkeit für Bibliotheken, hier einen Bestand aufzubauen. Zudem werden einige Spiele, die online gespielt werden, kostenlos angeboten. Das bedeutet, dass selbst das Argument bzw. Geschäftsmodell des freien Zugangs zu Inhalten durch die Bibliothek nicht mehr greift. Zum anderen ist Online-Ggaming ein elementarer Bestandteil der Gaming-Kultur. Das bedeutet, dass Bibliotheken trotz allem einen Weg finden müssen, mit diesem Thema zu arbeiten.

Nun wäre es natürlich das einfachste, die Bibliothek könnte wie in einem südkoreanischen Internetcafé 30 bis 40 internetfähige Gaming-PCs aufbauen und somit den Kunden die Möglichkeit geben, in der Bibliothek zu spielen. Dass Bibliotheken einen Zugang zum Internet bereitstellen müssen, ist sicherlich akzeptiert und ich hoffe sehr, dass es in wenigen Jahren in Deutschland keine Bibliothek mehr gibt, die nicht über einen Hochgeschwindigkeits-Internetanschluss inkl. kostenfreiem und zugleich frei zugänglichem WLAN für die Kunden verfügt. Allerdings wird es nicht möglich sein, die Bibliotheken in der Breite mit Gaming-PCs auszustatten. Zudem würde ein solches Konzept unglaublich viel Platz benötigen. Von der benötigten Energie und weiteren technischen und personellen Ressourcen ganz zu schweigen. Ganz anders sieht es aber aus, wenn man nur temporär etwas mit dem Thema Onlinegaming macht. Dann ist es durchaus möglich, auch dieses Genre in das Angebot der Bibliothek zu integrieren.

Es macht für eine Bibliothek wenig Sinn, einfach nur einen PC aufzustellen und darauf ein paar Spiel-Clients für Online-Games bzw. Browser zu installieren. Die Welt des Onlinegamings ist sehr facettenreich. Es wird also notwendig sein, eine Vorauswahl zu treffen und dies bedeutet: Sie müssen selbst spielen. Aber Spielen alleine wird natürlich nicht ausreichen. Auch in diesem Fall müssen Sie wieder überlegen, was Sie den Gamern anbieten können, was sie woanders nicht bekommen werden. 
Also was macht Ihren Event zu etwas ganz Besonderem? Eine Möglichkeit wäre, dass Sie auch hier einen Gaming-Wettbewerb durchführen. Wie das funktioniert, habe ich bereits beschrieben.

Für mich ein sehr interessanter Ansatz ist die Nutzung von Online-Games zur Vernetzung von Bibliotheken und ihrer Kunden. Die Idee ist, dass Sie in Ihrer Bibliothek für einen Zeitraum von z. B. zwei Wochen einen Gaming-PC installieren, auf dem man ein bestimmtes Spiel, welches einen Online-Multiplayer-Modus hat, anbieten. Hierfür können Sie jedes Spiel nutzen, welches einen Multiplayermodus hat und welches zudem eine Altersfreigabe ab 12 Jahren hat. Es gibt einige Spiele, die diese Anforderungen erfüllen. Das von mir im Folgenden beschriebene Spiel dient nur als Beispiel, um die Nutzungsmöglichkeiten des Online-Gamings zu erläutern. Eine Möglichkeit wäre das Spiel „Shoot Mania“. Es handelt sich dabei um einen Shooter, der allerdings ab 12 Jahren freigegeben ist. Das Spiel wird online gespielt, d.h. man braucht dafür eine kontinuierliche Internetverbindung. Die Aufgabe des Spielers ist sehr einfach: man muss mit seiner Spielfigur zusammen mit anderen das gegnerische Team suchen und besiegen. Nun würden also zu einem festen Zeitpunkt Spieler zu Ihnen in die Bibliothek kommen und gegen andere Bibliotheken online antreten. Insofern hat dieses Konzept eine sehr große Schnittmenge mit den beschriebenen Gaming-Wettbewerben.

Wenn Sie es wollen, können Sie den PC die ganze Zeit angeschaltet lassen. Dann können alle Bibliothekskunden, wann immer sie wollen, trainieren. Ich würde jedoch empfehlen, den Rechner nur dann die ganze Zeit angeschaltet zu lassen, wenn es sonst niemanden stört.

Natürlich können Sie die Vernetzung der Bibliotheken noch intensivieren. Hierzu müssten Sie nur in allen teilnehmenden Bibliotheken eine Webcam installieren, damit sich die Spieler gegenseitig sehen können.

Ziel dieses Projektes ist nicht nur das Spielen alleine. Es geht wie gesagt vielmehr um die Vernetzung der Spieler und der teilnehmenden Bibliotheken. Es ist deshalb auch möglich, zu bestimmten Zeitpunkten die Spieler zu vernetzen, ohne dass sie direkt spielen. Hierfür eignen sich Plattformen wie Skype oder Google-Hangout. Wichtig ist dabei, dass Sie dafür Accounts der Bibliothek nutzen.

Der Vorteil von vielen Online-Games liegt darin, dass sie zumeist nur geringe technische Ressourcen benötigen. Sie müssen dafür also keinen hochgerüsteten Gaming-PC organisieren. Sie benötigen allerdings einen offenen und schnellen Zugang zu Internet. Wenn Sie dies nicht ermöglichen können, ist für Sie Online-Gaming nicht umsetzbar.

\section{Zusammenfassung:}

Name der Veranstaltung: Online-Gaming

Zielsetzung: über die Nutzung Online-Gaming Bibliotheken und Ihre Gamer miteinander vernetzen

Benötigte Ressourcen: Computer inkl. Peripherie; Spiele, Raum, gegebenenfalls eine Webcam

Zeitrahmen: 2 Wochen (Vorbereitung ca. 10 Tage) 


\section{Spielewelten selber gestalten}

Bis jetzt haben wir darüber gesprochen, wie man Spiele in der Bibliothek spielen kann. Wir haben uns von einfachen Gaming-Events über Gaming-Wettbewerbe hin zum Thema Online-Gaming bewegt. Dies alles ist spannend und es gibt viel für Sie zu tun. Aber Gaming bedeutet bei weitem nicht nur das Spielen von Computerspielen. Gaming bedeutet auch unglaubliche Kreativität. In diesem Abschnitt geht es um die Möglichkeit, mit Games zu arbeiten und dabei nicht nur zu spielen.

Ich weiß nicht, wie es Ihnen geht, aber ich habe seit den ersten Tagen, an denen ich Computerspiele spielte, überlegt, dass ich manche Spielewelt gerne verändern bzw. neu gestalten würde. Während dies zu meiner Kinderzeit bzw. Jugend nicht möglich war, kann man dies heute bei immer mehr Spielen tun. Was am Anfang ein kleines Addon war, ist heute eine große Möglichkeit, um die Kreativität und die $\mathrm{Zu}$ sammenarbeit von Gamern in der Bibliothek zu fördern.

Es gibt eine Vielzahl von Spielen, bei denen die Spieler die Spielewelt verändern bzw. komplett neu gestalten können. Und genau diese Möglichkeit sollten sich Bibliotheken zu Nutze machen. Im Folgenden möchte ich anhand von drei Beispielen aufzeigen, was dies im Detail bedeutet. Es handelt sich dabei um die Spiele „Trackmania“, „Portal 2“ und „Minecaft“.

Beginnen wir mit „Trackmania“. Dieses Spiel ist ein sogenanntes Rennspiel. Es geht also um Autorennen. Das besondere an „Trackmania“ ist nicht das Rennen an sich, sondern die Möglichkeit, eigene Rennstrecken zu bauen. Hierfür gibt es spezielle Editoren, die einfach zu bedienen sind. Es entstehen individuelle Rennstrecken, auf denen man mit seinem Wagen Rennen fahren kann. Besonders interessant ist dabei, dass man die Rennstrecken mit anderen teilen kann. Man kann andere Spieler auf die jeweilige Strecke einladen und dann gegeneinander fahren.

Ein vergleichbares Modell finden wir bei dem Puzzle „Portal 2“. In diesem Spiel geht es darum, sich in einem dreidimensionalen Raum über verschiedene Hindernisse von Punkt A nach Punkt B zu bewegen. Das Spiel spielt man aus der First-Person-Perspektive, es wirkt also wie ein Shooter. Man hat sogar eine Art Kanone in der Hand, mit der man schießen kann. Mit der Kanone kann man Portale erstellen. Schießt man mit der linken Maustaste auf eine Wand, entsteht in der Wand ein Loch, welches den Eingang zum Portal darstellt. Schießt man mit der rechten Maustaste, so entsteht ein weiteres Loch, welches den Ausgang des Portals darstellt. Man kann nun durch den Eingang des Portals gehen und kommt dann am Ausgang des Portals an. Auf diese Art und Weise kann man die jeweiligen Hindernisse überwinden. Portal ist ein beeindruckendes Spiel und es macht sehr großen Spaß. Es ist zudem gewaltfrei. Auch bei Portal 2 kann ich individuelle Spielwelten erstellen und mit anderen teilen. Und auch für dieses Spiel gibt es einen einfach zu bedienenden Editor.

Kommen wir schließlich zu Minecraft. Dieses Spiel habe ich ebenfalls schon beschrieben. Es handelt sich kurz gesagt um einen riesigen virtuellen Baukasten, bei dem die Spieler quadratische Blöcke bewegen und verändern können, um u. a. riesige Gebäude entstehen zu lassen. Minecraft ist vor allem aufgrund der durch die Spieler entwickelten Gebäude berühmt geworden. Das Spiel ist ideal für kreative Gamer, die eine eigene Spielewelt entstehen lassen möchten. Schon die Möglichkeit, dass man alleine die jeweilige Spielewelt durch seine Gebäude gestalten kann, ist beeindruckend. Es ist aber ebenso möglich, eine eigene Spielewelt zu erstellen, die dann als Spielfeld genutzt werden kann.

Was alle drei genannten Spiele vereint, ist die Tatsache, dass man sie nicht nur spielen, sondern auch Spielewelten für andere Spieler erstellen kann. Diese Erstel- 
lung einer Spielewelt ist also elementarer Bestandteil des Spiels. Für Bibliotheken ergeben sich daraus resultierend eine Vielzahl an Möglichkeiten.

Die Bibliothek sollte der Ort sein, an dem die Spieler ihre Spielewelten erstellen, teilen und vermitteln können. Lassen Sie mich zuerst erklären, warum diese Option für Bibliotheken so wichtig sein könnte. Wie bereits am Anfang des Buches besprochen, ist Gaming mehr als nur Spielen. Gaming kann für Bibliotheken die Möglichkeit sein, eine Vielzahl an Kompetenzen zu vermitteln und zu fördern. Dazu gehört auch die Nutzung moderner Komunikations- und Medientechnologien. Der Computer ist heute aus dem beruflichen und privaten Leben nicht mehr wegzudenken. Computerspiele sind für viele Menschen der erste Kontakt mit einem Computer. Darüber hinaus können Spiele, in denen Spieler eine eigene dreidimensionale Welt erschaffen, die Kreativität fördern. Aber das ist noch längst nicht alles. Diese Spiele können die Bibliothek zu einem Lern- und Spielort machen. Natürlich braucht man keine Bibliothek, um Zugang zu Spielen zu haben bzw. spielen zu können. Bibliotheken sind also nicht per definitionem Spielorte. Sie sind auch nicht per definitionem Lern- oder Leseorte. Ganz egal was Bibliotheken auch tun möchten, sie müssen sich immer überlegen, was ihr Angebot einzigartig macht.

Nun haben Sie verschiedene Möglichkeiten, mit den „Gamer created Enviroments“ zu arbeiten. Am einfachsten ist es natürlich, den Spielern zu ermöglichen, in der Bibliothek eine eigene Spielewelt zu kreieren. Aber das reicht m.E. nicht aus. Machen Sie aus der Bibliothek einen Ort der „großen Welten-Schöpfer“. Am besten wäre es, wenn Sie den Spielern die Möglichkeit geben, vor einem großen Publikum die Spielewelten zu kreieren und zu präsentieren. Am besten legen Sie einen Zeitraum fest, in dem die Spielewelten erschaffen werden müssen. Hierfür brauchen Sie nur einen Beamer und eine Leinwand sowie einen guten Laptop und einen dazugehörigen Internetzugang. Nach zwei Wochen würde dann ein Abend oder ein Nachmittag stattfinden, bei dem die Spieler ihre erstellte Welt präsentieren.

Daran anschließend geht es natürlich um das Spielen. Wir wollen ja nicht nur bunte Welten anschauen. Wir wollen spielen. Hierfür werden alle interessierten Spieler in die jeweiligen Spielewelten online eingeladen. Vor allem bei Trackmania ist dies spannend, denn man kann nun einen Wettbewerb starten, bei dem die Spieler ihre Rennen auf den Strecken fahren, die von den Spielern entwickelt wurden.

Wenn Sie erste Erfahrungen mit diesem Bereich gemacht haben, können Sie dann den nächsten Schritt gehen: Einen Wettbewerb bzw. eine Kooperation mit anderen Bibliotheken. Das Grundmodell ist ähnlich dem schon beschriebenen. Auch hier haben die Spieler die Aufgabe, Spielewelten zu erstellen und diese dann zu präsentieren bzw. andere Spieler einzuladen, in den Spielewelten zu spielen. Der Unterschied ist der, dass alle Aktivitäten in verschiedenen Bibliotheken parallel stattfinden. Wichtiges Element ist dann die Vernetzung der Spieler untereinander.

Sie können natürlich auch bestimmte Themen oder Aufgaben vorgeben. So könnte man in Trackmania die Aufgabe verteilen, dass die Spieler reale Rennstrecken nachbauen sollen. Die einzelnen künstlich erstellten Rennstrecken würden dann den anderen Spielern oder Bibliotheken präsentiert werden. Nun müssen die Spieler in den anderen Bibliotheken die jeweiligen Rennstrecken erraten. Hierfür wird es mit an Sicherheit grenzender Wahrscheinlichkeit notwendig sein, dass die Spieler im Internet recherchieren, um die Formen der einzelnen Rennstrecken herauszufinden. Sie können als Bibliothek auf diesem Weg also auch Recherchekompetenz im Internet vermitteln. Ein ähnliches Modell würde auch bei „Portal 2“ umgesetzt werden. Hier ist nur die Aufgabe eine andere. Die Spieler, die die Spielewelt erschaffen, sollen ein möglichst kompliziertes Puzzle erstellen, während die anderen Spieler das Puzzle möglichst schnell lösen sollen. 
Es gibt eine Vielzahl an Spielen, die es dem Spieler ermöglichen, eine eigene Spielewelt zu erschaffen. Sie alle eignen sich hervorragend für die Nutzung in der Bibliothek. Wichtig ist dabei u.a., dass Sie das Spiele und alle beschriebenen Funktionen vorab getestet haben. Sie können die Arbeit mit diesen Spielen auch zu einem Teil des schon angesprochenen „Summer Games“ machen.

\section{Zusammenfassung:}

Name der Veranstaltung: Spielewelten selber gestalten Zielsetzung: Die Spieler sollen eigene Spielewelten erschaffen Benötigte Ressourcen: Computer inkl. Peripherie; Spiele, Raum, Internetzugang Zeitrahmen: 2 Wochen (Vorbereitung ca. 10 Tage)

\section{Wikis und Sekundärinformationen}

Gaming ist Lernen. Gaming ist auch das Sammeln, Erschließen, Vermitteln von Sekundärinformationen. Immer mehr Spiele sind so komplex, dass die Spieler früher oder später Sekundärinformationen benötigen. Dies gilt zwar nicht für alle Spiele, aber in immer mehr Spielen ist dies der Fall. Nun nutzen Gamer natürlich eine Vielzahl an Plattformen wie Wikis und Blogs und Twitter für die Gewinnung, Sammlung und Vermittlung der Informationen. Der Wiki von „World of Warcraft“ ist beispielsweise einer der größten Wikis der Welt.

Das besondere an der Gaming-Kultur ist dabei die Tatsache, dass aktives Teilen von Game-relevanten Informationen eine große Bedeutung hat. Dies hat aber weniger mit einer sozialen Denkweise der Spieler zu tun. Es geht vielmehr um Ruhm und Anerkennung der Community bzw. um die Chance, gegen gleichwertige Gegner anzutreten. Gamer halten Ihr Wissen also nicht zurück um beim nächsten Spiel alle anderen Teilnehmer schlagen zu können. Wenn man dauernd gewinnt und es zudem immer einfacher wird, wenn es also keine Herausforderung mehr gibt, wird das Spiel langweilig und man hört auf, es zu spielen. Gamer sind keine Wesen, die kontinuierlich von Erfolg zu Erfolg schweben. Sie suchen neue Herausforderungen und deshalb teilen sie ihr Wissen. Und dann gibt es da noch den Ruhm und die Ehre in der Community. Die Spieler, die besonders viele Informationen teilen, bekommen die Anerkennung der anderen. Diese Herangehensweise ist vielen klassischen Informations- und Wissenssystemen überlegen. Denn hier werden nicht nur moderne Plattformen genutzt, sondern es existiert eine Kultur des Teilens.

Nun ist dies alles kein Automatismus und nicht alle Gamer sind gleichzeitig Experten für die Vermittlung und Erschließung von komplexen Informationen. Jedoch wissen wir, dass beispielsweise die Fähigkeit, Werkzeuge wie Wikis, Facebook-Gruppen, Communities, Twitter etc. professionell zu nutzen, ein wesentliches Erfolgskriterium bei der Suche nach einem passenden Job sein wird. Wir wissen ebenso, dass wir in Deutschland einen erheblichen Nachholbedarf hinsichtlich der Schulung und Vermittlung von Know-how in der Nutzung digitaler Medien haben. Die meisten Schulen befinden sich noch in der Kreidezeit und in den Universitäten sieht es nicht besser aus. Gleichzeitig ist es immer schwerer, Menschen für das Lernen von solchen Werkzeugen zu begeistern. Hier kann Gaming helfen.

Wir wissen, dass das Sammeln, Erschließen und Teilen von Informationen im Bereich Gaming eine große Bedeutung hat. Wir wissen auch, dass bestimmte Communities bereits heute Tools wie Wikis und andere Plattformen für ihr Wissensma- 
nagement nutzen. Mein Vorschlag ist nun, die Vermittlung von Kompetenz in der Nutzung von Wikis und Co. mit dem Thema Gaming zu verbinden. Hierfür laden Sie Gamer in die Bibliothek ein, die dann bestimmte Spiele spielen sollen. Im Anschluss daran beginnen Sie alle zusammen mit dem Aufbau eines bibliothekseigenen Wikis, in dem dann wichtige Informationen zum jeweiligen Spiel gesammelt werden. Am spannendsten wäre es, wenn Sie einen Wiki erstellen, in dem alle Spiele behandelt werden, die Sie im Bestand Ihrer Bibliothek haben. Dies hätte zudem den Vorteil, dass Sie als Bibliothek zu allen Spielen Zusatzinformationen anbieten können. In diesem Wiki gäbe es immer zwei Bereiche. In dem ersten Bereich wird das jeweilige Spiel beschrieben. Der Leser erfährt, um was es in dem Spiel geht und wie man es spielt. Im zweiten Bereich werden durch die Spieler Zusatzinformationen zur Verfügung gestellt. Wenn man z.B. ein Rätsel gelöst hat oder eine Abkürzung gefunden hat, kann man dies im Wiki veröffentlichen.

Natürlich werden nicht alle Spieler mit einem Wiki oder anderen Online-Tools vertraut sein. Das bedeutet, dass die Bibliothek hier Schulungen anbieten muss. Sie bekommen also nicht nur spannende Informationen zu Games, welche Sie in Ihrem Bestand haben, Sie sind ebenso in der Lage, eine sehr wichtige Kompetenz zu vermitteln.

Wenn Sie mit dem Wiki schon eine Weile gearbeitet haben, sollten Sie ihn öffentlich zugänglich machen. Noch besser wäre es natürlich, wenn der Wiki gleich von Anfang an frei zugänglich ist. Jedoch fühlen sich manche Bibliotheksmitarbeiter, Eltern und auch Gamer sicherer, wenn so eine Plattform erstmal geschlossen ist. Aber irgendwann sollte der große Tag kommen, an dem der Wiki für alle zugänglich gemacht wird. Machen Sie am besten einen großen Event daraus. Laden Sie die Menschen ein, stellen Sie die Gamer vor, die an dem Wiki mitgearbeitet haben und dann wird offiziell und für alle sichtbar der Wiki nach außen geöffnet. In diesem Moment sollte der Gaming-Wiki zu Ihrer täglichen Arbeit gehören, d.h. er ist u.a. Bestandteil Ihres Informationsangebotes - vergleichbar mit dem OPAC und Datenbanken. Binden Sie den Wiki unbedingt in Ihre Webseite ein und sorgen Sie dafür, dass so viele Menschen wie möglich erfahren, dass es ihn gibt. Rufen Sie die Menschen in Ihrem Umfeld dazu auf, an dem Wiki auch weiterhin mit zu arbeiten und küren Sie einmal im Monat den Gaming-Wiki-Mitarbeiter des Monats. Dieser Mitarbeiter wird aufgrund seiner Aktivitäten im Wiki von einer Gruppe von Bibliotheksmitarbeitern in geheimer Runde ausgewählt.

Wenn Sie möchten, können Sie in einem nächsten Schritt den Wiki auch für Spiele öffnen, die nicht in Ihrem Bestand sind. Hierbei müssen Sie nur darauf aufpassen, dass keine jugendgefährdenden Inhalte veröffentlicht werden. Eine Option wäre es, dass alle neuen Wiki-Seiten mit unbekannten Games von Ihnen bereits vor der Veröffentlichung geprüft werden. Sollten Sie den von mir vorgeschlagenen „GamingBeirat" ins Leben gerufen haben, welchen ich im Abschnitt zum Bestand beschrieben habe, können Sie auch diesen Beirat mit der Kontrolle des Wikis beauftragen.

\section{Zusammenfassung:}

Name der Veranstaltung: Wikis und Sekundärinformationen zu Games Zielsetzung: Die Spieler sollen gemeinsam mit Ihnen einen Gaming-Wiki aufbauen. Benötigte Ressourcen: Computer inkl. Peripherie, Internetzugang, Wiki-Software Zeitrahmen: dauerhaft (Vorbereitung ca. 10 Tage) 


\section{Storytelling und Gaming}

Bis jetzt haben wir uns nur wenig mit den Geschichten befasst, welche in den Spielen erzählt werden. Wir Menschen lieben Geschichten. Wir sind umgeben von Geschichten und damals wie heute sind Menschen, die Geschichten erzählen können, gerne gesehene Gäste. Gaming und Geschichten sind eng miteinander verbunden - auch wenn es am Anfang schwer war, hinter den kleinen Pixeln eine Geschichte zu vermuten.

Storytelling ist Teil der Gaming-Kultur und dies gleich auf vielen Ebenen. Auf der einen Seite ist das die Geschichte des Gamings. Sie ist voll von Menschen die neue Ideen hatten und versuchten sie umzusetzen. Sie ist voll von Projekten, die scheiterten. Sie ist voll von Erfolgen und großen Persönlichkeiten. Und natürlich gibt es die Geschichten der Gamer. Da sind zudem die Geschichten, die in den Games erzählt werden. Und auch hier gibt es wieder mehrere Varianten. Es gibt Geschichten, die in den Games erfunden werden. In manchen Fällen ähneln diese Geschichten den Mythen und Märchen unserer Kultur. Da geht es um Drachen und Zauberer, um die Mafia, um Götter, um Krieg und Frieden etc. Dann gibt es Geschichten, die auf unserer Realität basieren. Dies können die jetzige Zeit, aber auch historische Begebenheiten sein. So erleben Sie z.B. Schlachten aus dem zweiten Weltkrieg neu oder Sie fahren Formel 1-Rennen etc. Und natürlich können wir auch einen Sprung in eine mögliche Zukunft wagen. Die Spiele werden immer durch Geschichten zusammengehalten. Das Besondere daran ist, dass die Spieler Teil der Geschichte werden. Und natürlich gibt es auch eine Verbindung zu anderen Medien und Kulturwelten. So gibt es immer mehr Bücher und Filme, die auf Computerspielen basieren. In anderen Fällen erzählen Computerspiele die Geschichte eines Films oder eines Buches weiter. Gaming und Storytelling gehören also zusammen. Warum also nutzen Bibliotheken diese Verbindung nicht für neue Projekte? Wie wäre es, wenn Bibliotheken die Orte werden, an denen neue Geschichten aus der Gamingwelt entstehen bzw. vorhandene gesammelt werden? Lassen Sie einen Gamer seine Geschichte erzählen, ganz egal, ob es sich um seine persönliche Geschichte handelt oder er einfach eine Geschichte aus einem Spiel erzählt oder neu erfindet. Diese Sammlung von Geschichten wäre einzigartig und nur in Ihrer Bibliothek zu finden - selbst wenn Sie sie im Internet teilen würden. Ich weiß, dass das alles etwas komisch klingen mag. Bibliotheken als Orte, an denen Geschichten entstehen? Bibliotheken als Schreiborte? Probieren Sie es aus! Ich wette, Sie werden überrascht sein, was Sie damit alles erreichen können.

Wie aber könnte man als Bibliothek mit diesen Geschichten arbeiten? Beginnen wir mit einer einfachen Übung:

\section{Level 1:}

Bitten Sie die Spieler bei ausgesuchten Spielen, die Geschichte zu erzählen, die in den Games erzählt wird. In manchen Fällen kann es sei, dass die Spieler gar nicht genau wissen, worum es bei diesen Spielen eigentlich wirklich geht. Also werden Sie z. B. im Internet recherchieren müssen. In diesem Moment können Sie den Gamern zeigen, wie man noch besser im Internet recherchiert. Und wenn Sie dabei mit den Spielern intensiv zusammenarbeiten, können Sie selbst einige spannende Plattformen kennenlernen, auf denen es um Gaming geht. Wenn Sie die passenden Informationen gefunden haben, geht es weiter mit dem nächsten Level: der Erweiterung der Geschichte. 


\section{Level 2:}

Nun haben wir also herausgefunden, um was es in dem Spiel eigentlich geht. Auf Basis dieses Wissens können nun neue Geschichten erfunden bzw. die vorhandenen weiterentwickelt werden. Die Teilnehmer bekommen also die Aufgabe, die Geschichte weiterzuschreiben. Sie können dabei verschiedene Vorgaben machen. So können Sie die minimale und maximale Länge festlegen. Sie können auch vorgeben, dass bestimmte Figuren auftauchen sollten etc. Versuchen Sie aber wenn möglich, die Vorgaben auf ein Mindestmaß zu beschränken. Es ist wichtig, dass die Teilnehmer ihrer Kreativität freien Lauf lassen.

\section{Level 3:}

\section{Der Gaming-Geschichten-Lese-Club}

Nun haben wir also eine große Zahl von Geschichten gesammelt. Was machen wir nun damit? Hier können wir einen alten Bekannten aus der Welt der Bibliotheksarbeit weiterentwickeln: den Sommer-Lese-Club. Sie kennen den Club sicherlich besser als ich, also erspare ich uns eine erneute Definition des Clubs. Der Club eignet sich hervorragend, um die von den Spielern geschriebenen Geschichten zu lesen und weiterzuerzählen. Es gibt hier zwei Möglichkeiten. Entweder, Sie integrieren diese Geschichten in den vorhandenen Sommer-Lese-Club oder aber in vergleichbare Projekte, oder aber Sie erstellen einen eigenen Club, der nur ein ähnliches Konzept verfolgt. Die Aufgaben der Teilnehmer sind einfach:

1. Die Teilnehmer sollen die Geschichten lesen und in Interviews wiedergeben.

2. Die Teilnehmer sollen die Geschichten bewerten.

3. Die Teilnehmer sollen die damit verbundenen Spiele spielen.

Aus diesen Aufgaben wird am Ende ein Abschlussevent, bei dem die Spiele gespielt und die Geschichten nochmals präsentiert und prämiert werden. Bei diesem Modell wird eine Vielzahl an Fähigkeiten geschult. Gaming ist aber nur der Ausgangspunkt. Das Modell eignet sich zudem für eine Vielzahl von Altersgruppen. Sie können es auch älteren bzw. erwachsenen Gamern anbieten.

Eine besondere Variante wäre ein Event speziell für ältere Gamer bzw. zusammen mit ihren Kindern, bei denen die Eltern ihren Kindern erzählen, wie sie früher Gamer waren bzw. welche Spiele sie gespielt haben. Man könnte auch gemischte Teams erstellen, bei denen die Geschichten von Eltern und ihren Kindern zusammen entwickelt und präsentiert werden.

\section{Zusammenfassung:}

Name der Veranstaltung: Storytelling

Zielsetzung: Die Spieler sollen sich mit den Geschichten hinter den Spielen beschäftigen bzw. eigene Geschichten entwickeln,

Benötigte Ressourcen: Computer inkl. Peripherie; Spiele, Raum, Internetzugang

Zeitrahmen: 4 Wochen (Vorbereitung ca. 10 Tage) 


\section{Bibcraft}

Kommen wir nun zu einem Projekt, bei dem ein Spiel zur Bühne eines Events wird: Bibcraft. Bibcraft ist ein Konzept, welches Kelvin Autenrieth aus Leipzig entwickelt hat und welches er nun in die Welt der Bibliotheken tragen wird. Ich habe Bibcraft mit in dieses Buch aufgenommen, weil es meiner Meinung nach sehr gut zeigt, was Bibliotheken im Bereich Gaming tun können und wie man Inhalte der Bibliothek mit Gaming vernetzen kann.

Bibcraft basiert auf dem Spiel „Minecraft“ bzw. es nutzt dieses Spiel. Minecraft habe ich schon beschrieben. Es ist vereinfacht ausgedrückt ein Spiel, bei dem man eine Spielewelt verändern bzw. in der Spielewelt Gebäude und Strukturen bauen kann. Man nennt dieses Spielgenre auch „Open-World“ und in diesem besonderen Fall wird der Spieler zum Schöpfer einzigartiger Welten und Strukturen. Das Besondere an diesem Spiel ist also die Möglichkeit, selber etwas zu erschaffen und genau hier setzt Bibcraft an. Erinnern Sie sich noch an die TV-Sendung „Die Montagsmaler“. Bei dieser Sendung ging es darum, dass man Begriffe auf einem Display zeichnete. Dabei durfte man keine Wörter benutzen. Die anderen Teilnehmer mussten nun erraten, was das gerade Gezeichnete bedeuten sollte. Es war also ein Quizspiel und es machte Spaß mitzuspielen und auch, sich das Ganze anzusehen. Damit man auch selbst raten konnte, wurde vorab angesagt, wann die Lösung im TV angezeigt wird. Man konnte in diesem Fall die Augen schließen und zu Hause mitraten.

Bibcraft ist letztlich eine erweiterte Variante der Montagsmaler. Die Teilnehmer bilden Teams, die gegeneinander antreten. Das Ziel ist, mittels Darstellungen in Minecraft Begriffe zu erraten. Aus jedem Team muss immer eine Person in Minecraft eine Struktur, eine Figur, ein Gebäude etc. erstellen, damit die anderen Teammitglieder diese erraten können. Dabei wird die Zeit gestoppt, die jedes Team für das Erraten der Begriffe benötigt. Das Team, welches die einzelnen Begriffe am schnellsten errät, gewinnt.

Um dieses Spiel erfolgreich spielen zu können, benötigt man verschiedene Fähigkeiten. Man muss in der Lage sein, mit Minecraft zu arbeiten. Hier können Sie den Teams anbieten, vorab Trainigscamps durchzuführen. Zudem muss man kreativ genug sein, Begriffe darstellen und auf der anderen Seite erraten zu können. Wichtig ist dabei, dass alle Teammitglieder irgendwann Begriffe darstellen müssen. Zudem müssen sie in der Lage sein im Team zu arbeiten. Diese Fähigkeit ist besonders wichtig. Es reicht nicht aus, das Spiel zu beherrschen, man muss ebenso einen Weg finden, wie man die einzelnen Inhalte so darstellt, dass alle Teammitglieder diese gut verstehen.

Wenn Sie möchten, können Sie daraus eine richtige Liga entwickeln. In diesem Fall sollten Sie aber zweigleisig fahren. Wenn Sie Teams aufbauen können, die sich intensiver mit dem Spiel auseinandersetzen möchten, müssen Sie diesen Teams unbedingt einen Raum zur Verfügung stellen. Die Bibliothek als Spielort wird erst dann erfolgreich sein, wenn nicht nur die finalen Events, sondern ebenso die Trainings und die Vorbereitungsmeetings der Teams bei Ihnen in der Bibliothek stattfinden. Gleichzeitig müssen Sie aber ein Einstiegsangebot entwickeln. Einmal im Monat sollte eine Bibcraft-Veranstaltung stattfinden, bei der alle Interessierten mitmachen können. An diesem Abend wird der Schwierigkeitsgrad gesenkt. Und vielleicht macht es an solche Tagen auch Sinn, dass man nicht im Spiel arbeiten muss, sondern einfach „nur“ zur Gruppe der Ratenden gehört.

Inhaltlich bietet Bibcraft für Sie viele Möglichkeiten oder anders ausgedrückt, Ihre Bibliothek ist für Bibcraft eine wahre Goldgrube. Egal, ob Sie eine öffentliche oder eine wissenschaftliche Bibliothek sind, Sie verfügen über Millionen Milliarden 
Inhalte. Ihre Bücher, Datenbanken, Info-Systeme sind voll von Dingen, die man in Bibcraft erraten bzw. bearbeiten könnte. Es geht also weniger um die Frage, wo Sie Fragen bzw. zu erratende Inhalte finden können, sondern vielmehr darum zu überlegen, für wen Sie dieses Projekt anbieten. Wenn Sie dieses Projekt z. B. als öffentliche Bibliothek anbieten und Ihre Teams aus Menschen unterschiedlichen Alters bestehen, dann können Sie alle mehr oder weniger bekannten Romane, Thriller, klassische Werke, aber auch Musik und Filme als Inhalte nutzen. Wenn Sie aber nur ein Spezialangebot für Musikbegeisterte anbieten möchten, dann würden Sie sich eben auf dieses Thema fokussieren. Gleiches gilt für wissenschaftliche Bibliotheken. Wenn Sie eine wissenschaftliche Bibliothek sind, dann haben Sie es z. B. mit Studenten zu tun, die bestimmte Themen lernen sollen. So können Sie speziell für diese Zielgruppe derartige Veranstaltungen anbieten.

Bibcraft können Sie aber noch weiter ausbauen. So können Sie bei erfahrenen Teams die Aufgabe stellen, noch intensiver mit dem Bestand der Bibliothek zu arbeiten. Wenn ein Team z. B. den Titel eines Buches erraten hat, dann kann es eine weitere Aufgabe sein, das Buch möglichst schnell im Bestand oder im Katalog zu finden.

Bibcraft ist ein wirklich spannendes Projekt und für Bibliotheken ist der Aufwand verhältnismäßig gering, denn die Ressourcen bezogen auf das Spiel sowie die Umsetzung bzw. Implementierung vor Ort können von dem Erfinder von Bibcraft eingekauft bzw. gemietet werden. Sie benötigen dann nur den Internetzugang, einen Beamer, Laptop etc. Es geht also um Ressourcen, die die weitaus meisten Bibliotheken besorgen können bzw. im Bestand haben.

Bibcraft ist wie bereits beschrieben ein Modell, welches die Spiele als Plattform nutzt, um damit etwas komplett anderes zu machen. Es gibt weitere Projekte wie z. B. die Aktivitäten der Initiative „Creative Gaming“. Auch hier werden Games als Basis genutzt, um damit völlig andere Dinge zu tun. So führt man z. B. ein Ballett in einem Ego-Shooter auf etc., ein anderes spannendes Projekt ist „This Spartans Live“. Dieses Projekt wurde von Gamern initiiert. Die Frage, mit der sich die Gamer beschäftigten, war die, was man z. B. mit einem Ego-Shooter noch alles machen könnte außer Schießen und Jagen. So kam man auf die Idee, im Ego-Shooter „Halo“ eine Talkshow zu entwickeln und als Video aufzunehmen. Wie in einer richtigen Talkshow gibt es auch hier Gäste und Interviews. Der Unterschied ist der, dass die jeweiligen Spielfiguren, die Avatare und nicht die Menschen zu sehen sind. Der Trick dahinter ist der, dass „Halo“ auch einen Multiplayermodus hat. Alle Menschen, die dieses Spiel im Multiplayermodus spielen, sehen die anderen Spielfiguren. Also hat man eine Person als Kamera umfunktioniert, d.h. diese Figur bewegt sich nicht. Die anderen Figuren bewegen sich nun im Blickfeld der ersten Figur und das, was die erste Figur sieht, wird als Video aufgezeichnet.

Auch hier hat man also das Spiel umfunktioniert bzw. es als Bühne für andere Aktivitäten benutzt. In all den genannten Beispielen, von „Bibcraft“ über „Creative Gaming“ bis hin zu „This Spartans Life“, stehen nicht mehr die Spiele im Vordergund, sondern das, was ich damit tue. Nun mögen Sie vielleicht einwenden, dass man ja dann die Spiele eigentlich gar nicht braucht und man alle Aktivitäten auch ohne Spiele durchführen könnte. Warum nicht die Montagsmaler mit einem iPad durchführen? Warum nicht ein echtes Ballett oder eine echte Talkshow entwickeln? Die Antwort ist ganz einfach: weil es dann nichts mehr mit Gaming zu tun hat. Damit fehlt Ihren Aktivitäten eine wichtige Schnittstelle zur Lebensrealität vieler Menschen. Und genau diese Schnittstelle ist es, die Sie brauchen, um mehr Menschen zu erreichen. 


\section{Zusammenfassung:}

Name der Veranstaltung: Bibcraft, Creative Gaming etc.

Zielsetzung: Nutzung von Games als Plattform für Aktivitäten in der Bibliothek

Benötigte Ressourcen: Computer, Internetzugang, Beamer, Leinwand (bei Bibcraft und vergleichbaren Projekten arbeiten Sie sehr oft mit externen Dienstleistern zusammen, die nicht nur für die Umsetzung, sondern auch für speziellen Ressourcen verantwortlich sind)

Zeitrahmen: dauerhaft (Vorbereitung ca. 10 Tage)

\section{Ingress}

Nun kommen wir zu einer Form von Spielen, bei denen Bibliotheken auf den ersten Blick überhaupt nichts beitragen können. Diese Spiele werden z.B. auf mobilen Devices wie Smartphones und Tablets gespielt. Das bedeutet, die Spiele stehen nur digital zur Verfügung und es gibt keine Möglichkeit, sie in den Bestand aufzunehmen. Des Weiteren werden diese Spiele nicht an einem Ort, sondern überall gespielt. Genauer gesagt muss der Spieler für jeden Schritt im Spiel an einen anderen Ort in der realen Welt kommen.

Das Beispiel, welches ich hier beschreiben möchte, heißt „Ingress“. Die Geschichte, die in diesem Spiel erzählt wird, ist schnell erzählt. Vor vielen Jahren wurde demnach bei Tests eine neue Materienform gefunden, die nun überall auf der Erde auftaucht. Besonders intensiv ist das Aufkommen in der Nähe von Portalen. Es gibt nicht viele Informationen über diese neue Materie, aber es zwei Gruppen von Menschen, die mit der Herausforderung unterschiedlich umgehen. Da gibt es zum einen die sogenannten „Erleuchteten“. Diese Gruppe geht davon aus, dass die neue Materie etwas Gutes ist und möchte dafür sorgen, dass die guten Eigenschaften der Materie allen Menschen zur Verfügung stehen. Auf der anderen Seite steht die Gruppe des sogenannten „Widerstands“. Diese Gruppe geht davon aus, dass die Materie für die Menschen gefährlich ist und möchte verhindern, dass sich die Materie weiter ausbreitet.

Das Spielprinzip ist verhältnismäßig einfach. Ingress ist eine App, die es nur für Android-Smartphones gibt. Dies liegt u.a. daran, dass der Anbieter des Spiels ein Tochterunternehmen von Google ist. Das Spiel basiert auf dem Kartendienst „Google Maps“. Allerdings haben die Karten eine andere Farbgebung bekommen. Man erkennt auf der Karte die Straßen und Wege, aber keine Gebäude oder andere Anlagen. Was man zudem sieht, sind die Portale. Diese Portale stehen in der Regel an besonderen Orten in der realen Welt wie z. B. historischen Gebäuden, Denkmälern etc. Das Spiel dreht sich im Wesentlichen um diese Portale. Diese sind entweder frei, oder aber sie werden von einer der beiden Fraktionen kontrolliert. Man kann diese Portale „hacken“, um bestimmte Items und Energie zu erhalten. Zudem kann man versuchen, die Portale einzunehmen bzw. gegen Feinde zu verteidigen, indem man ihre Verteidigungsanlagen ausbaut. Nun ist dieses Spiel ein „location-based Augmented Reality Game“. Das bedeutet, Sie können ein Portal nur dann bearbeiten, wenn Sie in der realen Welt vor Ort sind. Sie müssen sich also die ganze Zeit in der realen Welt bewegen.

Das Spiel ist aber nicht auf die beschriebenen Aktivitäten begrenzt. In der Regel können Sie kein feindliches Portal alleine übernehmen. Sie brauchen Mitstreiter, die ebenfalls in der realen Welt zusammenkommen, um dann die realen Orte aufzusuchen und dort die Portale zu hacken, zu schützen oder anzugreifen. Das Spiel vernetzt also Menschen in der realen Welt. Um die Kommunikation innerhalb der eigenen Gruppe zu verbessern, kann man sich zudem auf Google+ und in Chat-Gruppen 
miteinander vernetzen, um Fragen zu stellen und zu beantworten und um sich für Treffen in der realen Welt zu verabreden.

Das Spiel ist also nicht nur ein Spiel. Es fördert das vernetzte Denken der Spieler sowie deren Fähigkeiten in der Nutzung moderner Kommunikations- und Medientechnologien. Zudem entstehen neue Netzwerke von Menschen, die sich vielleicht sonst nie kennengelernt hätten.

Wie bereits gesagt können Bibliotheken mit Spielen wie Ingress auf den ersten Blick sehr wenig anfangen. Das Spiel passt nicht in den Bestand und man kann es kostenlos spielen. Auch als Spielort eignet sich die Bibliothek nicht, da man ja in der realen Welt von Ort zu Ort wandern muss, um die dortigen Portale zu finden bzw. dort aktiv zu werden. Und doch behaupte ich, dass Bibliotheken mit diesem Spiel sehr viel anfangen können.

Zum einen können Sie beantragen, dass Ihre Bibliothek ein eigenes Portal bekommt. Dies geht ganz einfach im Spiel, d.h. nur einer Ihrer Mitarbeiter mit einem Android-Smartphones müsste mit dem Spielen beginnen und am Standort Ihrer Bibliothek ein Portal beantragen. Keine Sorge: es ist kostenlos und Sie gehen keine Verpflichtungen ein. Es könnte aber dafür sorgen, dass viele Menschen den Weg zu Ihrer Bibliothek finden, und wenn Sie auch nur davor stehen bleiben und etwas mit ihren Smartphones tun. Sie können aber allen Spielern ein besonderes Angebot machen. So könnten Sie z.B. der Treffpunkt für die Spieler werden. Dafür müssen Sie nur ein Schild an die Tür hängen, auf dem steht, dass Sie alle Ingress-Spieler einladen, die Bibliothek zu betreten und z. B. sich dort aufzuhalten, um Strategien zu besprechen. Man könnte auch eine Ladestation für Smartphones anbieten. Sie müssen dafür nur die fünf am meisten vertretenen Android-Smartphones finden und dafür Ladegeräte kaufen. Dann brauchen Sie noch eine Steckdosenleiste und voila: Sie sind die ultimative Ingress-Profi-Gamer-Station.

Ich hoffe, dieses Beispiel zeigt Ihnen, wie Sie mit Games arbeiten können, die nicht Teil Ihres Bestandes und nicht Teil von Gaming-Veranstaltungen vor Ort werden können. Wenn die Bibliothek aber ein Gaming-Ort werden soll, dann müssen Sie alles versuchen, um auch exotische Angebote zu entwickeln. Ob sofort alle Ingress-Spieler zu Ihnen kommen werden, weiß ich nicht. Es ist aber so, dass solche Aktivitäten ein Statement pro Gaming sind, die sowohl nach außen als auch nach innen wirken. Dafür müssen Sie sich aber von der Idee verabschieden, man müsse als Ergebnis etwas mit Ihrem Bestand machen und es geht auch nicht darum, dass die Spieler bei Ihnen zum Lesen von Büchern animiert werden.

Wenn Sie es noch besser machen wollen, dann gründen Sie offene Ingress-Teams. Das bedeutet, Sie werden Teil der Spielewelt. Aber das ist nur etwas für die Bibliotheken, die alle anderen Aufgaben schon erfolgreich erledigt haben :-)

\section{Zusammenfassung:}

Name der Veranstaltung: Ingress

Zielsetzung: Die Bibliothek wird zum zentralen Spielort des Spiels.

Benötigte Ressourcen: Raum, Offenheit

Zeitrahmen: dauerhaft (Vorbereitung einmalig ca. 1 Tag) 


\section{Apps, Schnitzeljagden und die eigene Bibliothek}

Bevor Sie auch nur einen Moment daran zweifeln: Ich habe noch weitaus mehr Ideen und Vorschläge für die Nutzung von Gaming in Bibliotheken als jemals in dieses Buch passen würden. Alle hier gemachten Vorschläge sind quasi ein erster Einblick :-) Der nun folgende Vorschlag geht ebenfalls in die Richtung „mobiles Gaming“. Und auch hier haben Bibliotheken auf den ersten Blick keine Möglichkeiten der Implementierung bzw. Teilhabe. Wenn man jedoch genauer hinschaut, stellt man fest, dass Bibliotheken mit nahezu jedem Angebot etwas anfangen können.

Ein schönes Beispiel sind Apps für Smartphones, die den Nutzer spielerisch in der analogen Welt zu verschiedenen Orten führen. Diese Apps sind quasi interaktive Schnitzeljagden. Es gibt hier eine Vielzahl an Anbietern. Ein spannendes Beispiel ist „Actionbound“. Das besondere an Actionbound ist, dass man die Inhalte der App selber generieren kann. Man ist also nicht abhängig von vorgegebenen Inhalten. Also kann man diese App auch spielerisch verwenden und nun kommt die Bibliothek ins Spiel. Am Beispiel von zwei Nutzungsvarianten möchte ich die Optionen kurz beschreiben:

1. Das ultimative Bibliotheks-Geschichten-Spiel: Dabei geht es darum, eine Geschichte zu erzählen, die z. B. in Ihrer Stadt oder Ihrem Dorf spielt. Sie können hierfür als Basis Geschichten nutzen, die z.B. in Büchern aus Ihrem Bestand erzählt werden. Vielleicht gibt es aber auch historische Geschichten aus Ihrem Umfeld. In einem ersten Schritt müssten die Teilnehmer dann im Rahmen von Workshops gemeinsam eine Geschichte erfinden. Daran anschließend muss diese Geschichte in ein Spiel umgewandelt werden. Schließlich wird dieses Spiel nun auf die App übertragen. Nun können alle Interessierten das Spiel spielen und sich auf die Reise durch Ihre Stadt bzw. Ihr Dorf machen. Die Nutzung von Actionbound ist dabei sehr einfach. Sie müssen also nichts programmieren.

2. Das Erstsemester-Spiel einer Universitäts-Bibliothek: Es gibt viele mögliche Services für wissenschaftliche Bibliotheken, aber es gibt kaum einen Moment, an dem Sie näher an den Studierenden sind als in den ersten Tagen des Semesters. Gewiss, diese Studenten brauchen nicht sofort eine Bibliothekseinführung und müssen auch nicht gleich alle Datenbanken und Kataloge nutzen können. Aber diese Studenten brauchen Informationen. Und dabei geht es um all das, was sie in den ersten Wochen tun und kennenlernen müssen. Die Bibliothek kann hier zur zentralen Informationsstelle werden. Und wenn Sie z. B. einen großen Campus haben und zudem einige wichtige Orte in Ihrer Stadt kennen, dann wäre es doch spannend, Sie könnten diese Informationen auf spielerische Weise mittels einer App vermitteln, oder? Und genau dafür können Sie Angebote wie Actionbound nutzen.

Beide beschriebenen Varianten sind umsetzbar und beide Varianten kann man ausbauen. Sie müssen auch nicht sofort das ultimative Spiel bauen und natürlich sollen Sie auch weiterhin Ansprechpartner in der realen Welt sein. Und doch können Sie mit solchen Angeboten sehr viel erreichen. Zum einen haben Sie damit neue spannende Angebote erschaffen - und Sie müssen die App nicht selber programmieren. Sie ist ja schon da. Zum anderen lernen Sie als Bibliothek, wie man mobile Angebote wie Apps nutzt und wie man zudem daraus ein Spiel kreiert. Schließlich werden Sie zu einem Innovationsträger in Ihrem Umfeld und Sie sorgen dafür, dass die Menschen Sie als eine Institution wahrnehmen, die sehr viel mehr kann als Medien verleihen, Leseförderung und Co. 


\section{Zusammenfassung:}

Name der Veranstaltung: Mobile Games selber „bauen“ und nutzen

Zielsetzung: Die Spieler sollen gemeinsam mit Ihnen die Inhalte für eine App erstellen und daraus ein mobiles Game machen.

Benötigte Ressourcen: Computer inkl. Peripherie, Internetzugang

Zeitrahmen: dauerhaft (Vorbereitung ca. 5 Tage)

\section{Kudo - ich baue mir ein Computerspiel}

Jetzt wird es richtig spannend. Nun kommt nämlich der nächste Level. Ich hoffe Sie sind noch immer motiviert und haben schon erste Ideen für Gaming-Aktivitäten in der eigenen Bibliothek entwickelt? Bis jetzt haben wir uns mit schon vorhandenen Spielen und Plattformen beschäftigt. Nun gehen wir einen Schritt weiter und bauen unsere eigenen Spiele! Stellen Sie sich vor, Ihre Bibliothek wäre der Ort, an dem Menschen zusammenkommen, um Computerspiele zu entwickeln und diese dann mit anderen zu spielen. Wäre das nicht wirklich cool? Nun werden Sie wahrscheinlich davon ausgehen, dass man dafür komplexe Programmiersprachen beherrschen muss und Wochen, ja Monate Zeit braucht. Aber dem ist nicht so. Am Beispiel der Software „Kudo“ möchte ich Ihnen zeigen, wie Ihre Bibliothek zu einem Raum für kreative NachwuchsGames-Entwickler wird.

Die Idee von Kudo ist verhältnismäßig einfach. Die von Microsoft entwickelte Software soll es letztlich jedem Menschen ermöglichen, sein eigenes Computerspiel zu entwickeln, ohne dabei Informatik studieren zu müssen. Hierfür wurde ein Editor erschaffen, der mittels Symbolen die Programmierung eines Spiels ermöglicht. Das Konzept ist für Kinder gedacht, was bedeutet, dass es wirklich einfach zu verstehen und zu erlernen ist. Um etwas zu programmieren, braucht man in Kudo zwei Parameter. Die Aktion und das, was sie hervorruft. Dies geschieht über eine Auswahl an Symbolen, die die jeweiligen Aktivitäten beschreiben. Dabei ist alles sofort sichtbar, d.h. man arbeitet direkt in der Spielewelt und kann natürlich auch deren Struktur verändern. Die Eingabe erfolgt über einen XBOX-Controller, man braucht also keine Tastatur und auch keine Kenntnisse über Tastaturbefehle bzw. einen Programmiercode und man kann das jeweils entwickelte sofort sehen. Damit aber nicht genug. Die Spiele funktionieren auf dem PC und auf der XBOX 360. Darüber hinaus kann man die Spiele auch in einer Community teilen und testen lassen. Es entsteht also eine stetig wachsende Community an jungen Nachwuchs-Games-Entwicklern und für Bibliotheken ist dies ein geniales Angebot.

Zuerst können Sie auch hier wieder der Ort für Gaming sein. Dieses Mal geht es aber primär nicht um das Spielen, sondern um das Programmieren. Ihre Bibliothek wird also zum Kreativzentrum. Sie sind der Ort, an dem Menschen zusammenkommen, um neue Spiele zu erschaffen. Sie können dies wunderbar an bereits vorhandene Events andocken. So wäre dies sicherlich auch als Erweiterung des SommerLese-Clubs interessant. Und natürlich gehört so etwas in die Summer Games Ihrer Bibliothek. Aber das ist längst noch nicht alles. Denn diese Spiele, die bei Ihnen entwickelt werden, werden auch Teil Ihres Bestandes. Zwar kann man Sie nicht auf DVD brennen und verleihen, aber man kann den Zugang zu ihnen schaffen. Es ist so wie bei den eBooks. Auch dort geht es letztlich nur darum, einen Zugang zu schaffen. Sie haben dann aber Unikate in Ihrem Bestand! Diese Spiele sind bei Ihnen in der Bibliothek entstanden. Das ist so als wäre Goethe oder Shakespeare zu Ihnen in die Bibliothek gekommen, um nur bei Ihnen ein Werk zu verfassen. Dieser Content ist 
einzigartig und diejenigen, die diese Spiele entwickelt haben, werden stolz darauf sein, dass andere Menschen ihre Spiele spielen.

Damit kommen wir auch schon zum nächsten wichtigen Punkt. Es ist nämlich wichtig, dass die Spiele nicht nur bei Ihnen entstehen, sondern dass man sie auch bei Ihnen spielen kann. Also sollten Sie die Spiele in einem besonderen Event den anderen Bibliothekskunden vorstellen. So wie Sie vielleicht auch Lesungen mit Autoren anbieten, würden Sie jetzt Gaming-Abende haben, bei denen die Entwickler ihre Spiele vorstellen und jeder diese Spiele ausprobieren kann. Am Ende sollten diejenigen, die die Spiele ausprobiert haben, diese auch bewerten. Und so wird einmal im Jahr in Ihrer Bibliothek der Kudo-Games-Entwickler des Jahres gekürt.

Und natürlich können Sie auch hier wieder mit anderen Bibliotheken sowie anderen Kultur- und Bildungsinstitutionen kooperieren und ein Netzwerk aufbauen. Hier würden dann irgendwann in 20 oder 30 Bibliotheken Spiele entstehen und diese Spiele wären auch in allen teilnehmenden Bibliotheken spielbar.

\section{Zusammenfassung:}

Name der Veranstaltung: Kudo - Gamesentwicklung

Zielsetzung: Die Spieler sollen gemeinsam mit Ihnen mittels Kudo ein Spiel entwickeln. Benötigte Ressourcen: Computer inkl. Peripherie, Internetzugang, XBOX360, KudoSoftware

Zeitrahmen: Immer eine Woche (Vorbereitung ca. 3 Tage)

\section{Let's Play - Gaming-Videos in der Bibliothek drehen}

Wie ich bereits des Öfteren erwähnt habe, ist Gaming mehr als nur sinnlose Zeitverschwendung. In den allermeisten Fällen kommt ein Spieler irgendwann an einen Punkt, an dem er im Spiel nicht weiterkommt. Er braucht Hilfe von anderen Spielern. Und je komplexer ein Spiel ist, desto komplexer muss auch die Hilfe sein. So oder so werden aber weitere Informationen benötigt und es gibt hierzu kaum klassische Literatur. Und trotzdem ist es eigentlich kein Problem, sich über bestimmte Problemfelder Informationen zu beschaffen. Dies alles geschieht im Web. Es gibt unglaublich viele Webseiten, Wikis, Blogs, Foren etc., die sich mit bestimmten Spielen befassen. Und dabei wird das jeweilige Spiel nicht etwa oberflächlich behandelt. Die Spieler versuchen vielmehr alle möglichen Details und Herausforderungen zu finden, zu lösen und die Lösung anderen Gamern zur Verfügung zu stellen. Das aktive Teilen von Informationen ist ein elementarer Bestandteil der Gaming-Kultur und deshalb sind auch viele Gamer bzw. deren Informationssysteme den bibliothekarischen Angeboten weit voraus. Mailing-Listen wie Inetbib oder Forumoeb sind für Bibliotheken sicherlich ein Gewinn. Aus Sicht eines Gamers sind sie aber nicht ausreichend.

Nun findet die Vermittlung des Wissens zu den einzelnen Spielen nicht nur in Textform z.B. in Wikis oder Blogs statt. Es gibt auch Videos zu Spielen und damit meine ich nicht offizielle Trailer, sondern die sogenannten Let's Play-Videos.

Die Idee der Let's Play-Videos ist wie so oft sehr einfach. Ein Gamer spielt ein Spiel und nimmt dies auf Video auf. Dabei kommentiert er das, was gerade im Spiel passiert. Es ist eine Mischung aus Anleitung, persönlichem Statement und Entertainment. Natürlich spielt man ein Spiel nicht exakt so durch, wie es in dem Video beschrieben wird, aber man kann sehr viel lernen. Und es macht Spaß zuzusehen, da einige Kommentare der Spieler wirklich interessant sind. Es entsteht also eine neue 
Form des Zuschauens. Die Videos informieren und unterhalten und dies ist auch notwendig, denn manche Spiele dauern mehrere Stunden und auch die dabei entstehenden Videos sind so lang.

Für Bibliotheken sind Let‘s Play-Videos aus zwei Gründen interessant. Zum einen geht es wieder um den Bestand. Bibliotheken erleben den Wandel hin zu einer digitalen Gesellschaft hautnah. Es ist ein Wandel, der das Konzept bzw. Geschäftsmodell einer Bibliothek infrage stellt. Die Idee, Bibliotheken könnten die Masse der Informationen und Inhalte überblicken, strukturieren oder gar eine Vorauswahl treffen ist heute nicht mehr möglich. In bestimmten Bereichen, vor allem in wissenschaftlichen Bibliotheken, wenn es darum geht, einer ganz speziellen Zielgruppe Informationen zur Verfügung zu stellen, die auch nur in überschaubarer Größe angeboten werden, ist diese Modell vielleicht noch umsetzbar. Für öffentliche Bibliotheken und zukünftig für einen relevanten Bereich in den wissenschaftlichen Bibliotheken hat dieses Modell aber keine Zukunft mehr. Gerade die öffentlichen Bibliotheken erleben nun, dass sie nicht mehr ansatzweise in der Lage sind, die täglich ja stündlich zur Verfügung gestellten Informationen zu verarbeiten. Zudem haben wir es mit einer Vielzahl an relevanten Medien zu tun, die nicht mehr in einer Bibliothek verortbar sind. Dazu gehören auch und vor allem alle frei zugänglichen und zugleich kostenlosen Inhalte in Blogs, Wikis, Facebook, Flickr, Twitter etc. Die einzig sinnvolle Strategie ist nun, diese digitalen Inhalte trotzdem zum Bestand der Bibliothek zu erklären. Und das bedeutet auch, dass alle Youtube-Videos zum Video-Bestand einer Bibliothek gehören. Zwar kann man diese nicht ausleihen, aber Bibliotheken können Sammlungen erstellen, Informationen und Recherchedienste anbieten, selbst Videos drehen etc. Alle diese Dinge sind möglich und sollten auch von Bibliotheken umgesetzt werden. Wenn es also ein Let's Play-Video zu einem Spiel gibt, welches die Bibliothek im Bestand hat, so sollte die Bibliothek auf dieses Video hinweisen, wenn ein Kunde dieses Spiel ausleiht. Sie sollte zudem wissen, ob und wenn ja, wo es Let's Play-Videos zu den Spielen gibt, die gerade aktuell sind. Dies ist auch dann sinnvoll, wenn die Bibliothek das Spiel gar nicht im Bestand hat. Sie merken sicherlich, dass Ihr klassischer Bestand in diesem Bereich immer weniger Bedeutung hat und ich habe dieses Phänomen ja auch bereits beschrieben. Aber es geht nicht nur um die Entwicklung neuer professioneller Auskunftsdienste durch Einbeziehung von Online-Medien. Es geht auch darum, selbst in diesem Bereich aktiv zu werden und dies möchte ich nun beschreiben:

Bibliotheken sollten Orte sein, an denen Let‘s Play-Videos entstehen. Natürlich ist dies mit Aufwand verbunden. Sie benötigen einen PC, auf dem man auch spielen kann. Sie benötigen zudem eine spezielle Recordingsoftware, die den Desktop aufnehmen kann. Dann brauchen Sie noch ein Headset und Ihrer Gaming-VideoBibliothekskarriere steht nichts mehr im Wege. Wie bei allen anderen Projekten auch, benötigen Sie auch hier Geduld und Zeit. Und natürlich wird nicht alles klappen, aber Sie können mit diesem Angebot eine Vielzahl an Menschen erreichen. Und nebenbei vermitteln Sie eine Vielzahl an wichtigen und spannenden Kompetenzen:

1. Sie vermitteln die Kompetenz mit Technologien wie einer Spezialsoftware für das Mitschneiden von Spielen umzugehen.

2. Sie vermitteln Social Media-Kompetenz, denn YouTube ist eine Social MediaPlattform. Die Spieler lernen nicht nur die Funktion, sondern auch, dass YouTube mehr ist als eine Spaß-Plattform.

3. Sie vermitteln auch die Erkenntnis, dass Gaming mehr ist als nur einfaches Spielen. 
Auch in diesem Fall entsteht mehr als nur ein Video. Dieses Video ist ebenfalls Teil Ihres Bestandes und es ist bei Ihnen entstanden. Es ist ein weiteres Alleinstellungsmerkmal. Und Sie können dieses Video nutzen, um einen zusätzlichen Auskunfts-Service anzubieten. Die Bibliothek wird auf diesem Weg weiteres Know-how aufbauen. Die Fähigkeit, Videos zu drehen und bei YouTube einzustellen wird die Bibliothek auch in anderen Bereichen nutzen können.

Natürlich ist es auch hier sinnvoll, die Videos im Rahmen einer Veranstaltung vorzustellen. Sie können dabei andere Gamer einladen, aber natürlich auch die Eltern der Gamer - vor allem, wenn es junge Gamer sind, die die Videos erstellt haben. Wenn Sie eine solche Veranstaltung durchführen, sollte man auch die Spiele vor Ort spielen können. Wenn alles gut läuft, können Sie weitere Menschen in Ihrem Umfeld finden, die ebenfalls Gamer sind und/oder Let‘s Play-Videos gedreht haben.

\section{Zusammenfassung:}

Name der Veranstaltung: Let's Play-Videos erstellen

Zielsetzung: Die Spieler sollen gemeinsam mit Ihnen Let's Play-Videos erstellen. Benötigte Ressourcen: Computer inkl. Peripherie, Internetzugang, Recordingssoftware Zeitrahmen: Immer eine Woche (Vorbereitung ca. 3 Tage)

\section{Alternate Reality Games von und mit der Bibliothek}

Stellen Sie sich vor, Sie könnten die Bibliothek, die digitale Welt und vielleicht noch einige weitere Orte in Ihrer Stadt bzw. Ihrem Dorf zu einer Spielewelt umfunktionieren? Wie wäre das? Nun werden Sie sagen, dass dies nicht möglich ist, ich aber sage Ihnen: Es geht und es macht großen Spaß. Das Thema „Alternate Reality Games“ habe ich schon behandelt. Es sind keine Spiele, die man mit einer Konsole oder einem Computer spielt. Es geht vielmehr um die Idee, das Spiel in die Lebensrealität der Menschen zu holen. Diese Spiele erzählen Geschichten bzw. die Spieler werden Teil der jeweiligen Geschichte. Als Plattformen dienen alle möglichen Kommunikationsmedien wie z. B. Social Media, eMail, Telefon. Zudem gibt es immer wieder Aktivitäten in der realen Welt, also von realen Menschen an realen Orten. Die Spiele schaffen folglich für den Spielenden eine alternative Realität. Die Nutzungsmöglichkeit sind vielfältig.

Auch Bibliotheken können mit Alternate Reality Games arbeiten. Und es gibt sogar zwei Möglichkeiten:

1. Es wird von einer kleinen Gruppe ein Alternate Reality Game entwickelt, welches dann in der Bibliothek spielt und die Bibliothekskunden miteinbezieht. In diesem Fall könnte beispielsweise eine Geschichte aus einem Buch im Bestand der Bibliothek als Vorlage dienen. Man könnte auch regionale historische Themen aufarbeiten. Oder aber man lässt die Bibliothek und Ihre Mitarbeiter zum Inhalt der Geschichte werden.

2. Die Entwicklung eines Alternate Reality Games ist Teil eines Projektes in der Bibliothek. In diesem Fall gibt es eine größere Gruppe bestehend aus Bibliotheksmitarbeitern und natürlich Bibliothekskunden, die gemeinsam ein oder mehrere Alternate Realiy Games entwickeln und dann auch umsetzen.

Beide Varianten sind spannend und eignen sich auch für die Bibliotheken, die noch immer Schwierigkeiten mit einer gut funktionierenden digitalen Infrastruktur haben. 
Allerdings erfordert die Umsetzung eines solches Spiels eine Vielzahl an Fähigkeiten. Dazu gehört z. B. auch eine tiefgreifende Kompetenz im Bereich Storytelling über Soziale Medien. Ebenso wichtig ist die Bereitschaft, die Bibliothek als Bühne zu sehen, auf der der weitaus größte Teil des Spiels stattfindet. Sie werden am Anfang am besten in kleinen Schritten aktiv sein. Sie müssen nicht sofort das ultimative Game entwickeln bzw. anbieten. Es ist viel einfacher, wenn Sie sich in kleinen Schritten an das Thema heranwagen. Versuchen Sie z. B. auf Ihren Onlineplattformen eine Geschichte zu erzählen, die inhaltlich zu Ihrer Bibliothek passt und die zudem in Teilen in der Bibliothek spielt. Natürlich ist dies nicht ein komplettes Alternate Reality Game, aber es ist eine Möglichkeit, sich langsam dem Thema zu nähern.

\section{Zusammenfassung:}

Name der Veranstaltung: Alternate Reality Games

Zielsetzung: Die Bibliothek als Anbieter eines solchen Games oder als Ort, an dem ein solches Game entwickelt wird

Benötigte Ressourcen: Computer inkl. Peripherie

Zeitrahmen: Immer eine Woche (Vorbereitung ca. 3 Tage)

\section{Retro-Gaming}

Retro-Gaming ist mit Sicherheit eines der spannendsten Themen in Bibliotheken, wobei dies mit Sicherheit vor allem ein Thema für öffentliche Bibliotheken ist. Allerdings habe ich in den USA erleben dürfen, dass auch an Universitäten riesige GamingArchive bestehen und die Bibliothek hier integriert ist. Aber natürlich ist mir klar, dass das Thema Retro-Gaming an deutschen Universitäts- und Fachhochschulbibliotheken vorerst keine Rolle spielen wird, auch wenn diese Bibliotheken damit einiges an Potential verschenken.

Aber kommen wir nun zum Thema Retro-Gaming. Erinnern Sie sich noch an den Beginn dieses Buches? Dort habe ich über meine eigene Gaming-Geschichte geschrieben. Und ich bin immerhin Ende 30 (Anfang 40 hört sich nicht so gut an wie Ende 30). Nun gibt es tausende Frauen und Männer die älter sind als 28 und eine eigene Gaming-Vergangenheit haben. Wenn Sie als Bibliothek verstanden haben, dass Gaming nicht nur ein Thema für Kinder, Jugendliche und junge Erwachsene ist, und wenn Sie auch für diese ältere Zielgruppe als Bibliothek relevant sein wollen, dann kommen Sie an dem Thema Retro-Gaming nicht vorbei. In einem ersten Schritt geht es natürlich wieder um Gaming-Events. Das bedeutet, Sie ermöglichen das Spielen von Retro-Games in Ihrer Bibliothek. Nun werden Sie mit Sicherheit einwenden, dass Sie die dafür notwendige Hardware nicht haben und dies deshalb gar nicht möglich ist. Und natürlich haben Sie damit auch Recht, aber es gibt hierfür zwei Lösungen:

1. Sie nutzen sogenannte Emulatoren bzw. adaptierte Spiele. Heute bekommt man überall die Spiele-Klassiker auch für den PC und Konsolen. Pac-Man, DonkeyKong und Co sind also auch auf heutigen Systemen spielbar.

2. Sie nutzen die Original-Konsolen. Es ist heute noch ohne weiteres möglich, alte Konsolen für verhältnismäßig wenig Geld zu kaufen. Ich habe für die Vorbereitung auf dieses Buch eine Nintendo NES für 40,- $€$ gekauft und das System ist kaum gebraucht. Auch Software können Sie noch immer in verschiedenen Online-Stores kaufen. 
Beide Varianten sind möglich und natürlich auch kombinierbar. Ich würde zudem empfehlen, in Ihrem Umfeld nach Hard- und Software zu fragen. Auf vielen Dachböden liegen noch viele Retro-Gaming-Schätze begraben. Ein kontinuierlich durchgeführter Aufruf kann hier wahre Wunder wirken. Am besten ist es, wenn Sie versuchen, eine eigene Sammlung aufzubauen. Der Aufwand ist geringer als es scheint. Das Einzige, was Sie tun müssen, ist kommunizieren und ausprobieren, ob die zur Verfügung gestellte Hard- und Software auch funktioniert.

Wenn Ihnen einige Geräte und die dazugehörige Software zur Verfügung stehen, sollten Sie einen Retro-Gaming-Tag veranstalten. Laden Sie die Menschen ein, bei Ihnen die Retro-Games zu spielen. Erstellen Sie zudem einen Retro-Games-Wiki und veranstalten Sie parallel, also am selben Tag, einen Gaming-Flohmarkt. Sie können mit den vorhandenen Konsolen und Spielen auch einen Parcours erstellen, den dann die Teilnehmer durchlaufen müssen. An einem solchen Tag sollten Sie auch Filme zeigen, die sich „damals“ mit dem Thema Gaming auseinandergesetzt haben. Hierzu gehören z. B. „Tron“ oder „Wargames“.

Sie können aber noch mehr tun. Laden Sie z. B. Eltern mit ihren Kindern ein und bitten Sie die Kinder, in der Bibliothek mit einer Videokamera ihre Eltern zum Thema Gaming zu interviewen. Dabei sollte es vor allem um die Gaming-Geschichte bzw. die Gaming-Kultur der Eltern gehen. Interessant wäre natürlich auch, im Anschluss daran die Eltern die Kinder interviewen zu lassen.

Wenn alles gut läuft, dann werden Sie in Zukunft sowohl die Erwachsenen als auch deren Kinder als Gaming-Kunden begrüßen können. Der Austausch der Generationen miteinander über die Gaming-Kultur ist etwas, was Bibliotheken unbedingt unterstützen sollten. Auf diese Art und Weise entstehen völlig neue Netzwerke für die Bibliothek. Und Bibliotheken werden zu einem Kulturort.

\section{Zusammenfassung:}

Name der Veranstaltung: Retro-Gaming

Zielsetzung: Verschiedene Generationen werden durch Retro-Gaming vernetzt.

Benötigte Ressourcen: Computer und Konsolen inkl. Peripherie, Spezialsoftware, Retro-Konsolen und Retro-Games

Zeitrahmen: ein Tag (Vorbereitung ca. eine Woche)

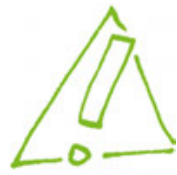

Achten Sie unbedingt auf die Video-Anschlüsse der Konsolen. Diese wurden in einer Zeit entwickelt, in der es noch keinen HDMI- und keinen DVI-Anschluss gab. In der Regel wurden sie über den Antennenanschluss an den Fernseher angeschlossen. Das bedeutet, dass Sie mit heutigen Monitoren nicht viel anfangen können. Wenn Sie einen Monitor nutzen möchten, muss dieser auch einen klassischen Antennenanschluss haben. Es gibt natürlich TV-Monitor-Kombinationen.

\section{Infoabende}

Ganz egal, wie erfolgreich Ihre Gaming-Aktivitäten auch sein mögen, Sie werden auf jeden Fall auch Informationsabende anbieten müssen. Diese Abende sind wichtig, denn an solchen Abenden können Sie für das Thema und Ihre Aktivitäten werben. Infoabende sind eine große Chance und ich weiß, dass viele Bibliotheken glauben, ein Infoabend wäre eine leichte Aufgabe, aber dem ist nicht so. Es gibt wenige Aktivitäten, bei denen Sie so viel falsch machen können wie bei einem Infoabend. Um die 
Gefahr negativer Konsequenzen minimieren zu können, sollten Sie ein paar wesentliche Dinge beachten.

1. Ein Infoabend ist keine Gefahren-Vermeidungs-Orgie. In vielen Fällen werden Infoabende zu Veranstaltungen, bei denen primär über mögliche Gefahren und dazugehörige Vermeidungsstrategien gesprochen wird. Natürlich gibt es, wie bei allen Medien - auch beim Buch -, Gefahren und Risiken, aber eine Bibliothek wird nie der Ort sein, an dem man sich mit Gaming befasst, damit Risiken vermieden werden. Das bedeutet nicht, dass Sie diesen Bereich totschweigen sollen. Aber er darf auf keinen Fall negativ besetzt werden.

2. Gamer sind Menschen. Das mag nun einfach klingen, aber versuchen Sie niemals, Gaming schlecht zu reden. Gaming ist nicht schlechter als das Lesen von Büchern. Und Gamer sind nicht schlechter als Buch-Liebhaber. Wobei wir wissen, dass Gamer auch lesen und umgekehrt.

3. Es gibt keine wertvollen und weniger wertvollen Spiele. Natürlich weiß ich, dass vor allem manche Eltern darüber nachdenken, welche Spiele man spielen sollte und welche nicht. Es heißt dann: „Wenn mein Kind schon spielt, dann soll es wenigstens etwas vernünftiges spielen“. Aber das Problem ist, dass die sog „vernünftigen“ Spiele in vielen Fällen keine guten Spiele sind, da hier nicht der Spielspaß, sondern ein Lernziel im Vordergrund steht. Verstehen Sie mich nicht falsch. Natürlich kann man sich über Spiele streiten und es ist von elementarer Bedeutung, dass Gamer nur die Spiele spielen, die für sie aufgrund ihres Alters geeignet sind. Aber lassen Sie bitte den Gamern Ihre Games. Eine Bibliothek ist keine ,+

4. Der Infoabend ist Ihr Abend! Es ist Ihre Möglichkeit, den Menschen Ihre Vision für die Nutzung von Games in der Bibliothek näher zu bringen. Es geht nicht darum, dass Sie sich rechtfertigen oder gar entschuldigen müssen. Sie können und Sie sollten selbstbewusst auftreten und klarstellen, dass Sie dieses Thema wollen. Fokussieren Sie sich in Ihren Präsentationen auf die Vorteile, die sich aus der Nutzung von Gaming ergeben. Gehen Sie auf das Thema „Gaming und Lernen“ ein. Versuchen Sie, das Thema positiv zu kommunizieren. Es muss den Menschen klarwerden, dass Sie Games für genauso relevant wie Bücher halten. Bereiten Sie sich gut vor. Wenn Sie dies alles beherzigen, dann sollten Sie als ein Programmpunkt auch auf die Gefahren und Risiken eingehen. Dies sollte aber von der Person vorgetragen werden, die die positiven Inhalte vermittelt hat. Sonst schaffen Sie einen inhaltlichen Konkurrenzkampf, der nicht notwendig ist. Vor allem: zeigen Sie auf, was Sie als Bibliothek schon alles im Bereich Gaming gemacht haben bzw. was Sie vorhaben. Zeigen Sie Beispiele von tollen Umsetzungen in Bibliotheken.

5. Laden Sie die Gamer ein. Es gibt nichts Schlimmeres als Reden über Menschen, ohne dass diese Menschen mit dabei sind. Machen Sie es nicht so wie auf den Bibliothekartagen. Auch dort redet man über die Menschen, aber nicht mit ihnen. Wenn Sie in Ihrer Bibliothek etwas zum Thema Gaming machen wollen, wenn Sie darüber informieren wollen, dann tun Sie dies am besten mit den schon vorhandenen Gamern zusammen. Und wenn Sie viele junge Gamer haben, dann müssen Sie eben den Infoabend früher durchführen.

6. Bauen Sie die Spiele auf. Die Teilnehmer sollen unbedingt spielen dürfen. Die persönliche Erfahrung ist wichtig. Wenn möglich sollten junge Gamer den Besuchern die Spiele erklären bzw. vorführen. Machen Sie aus der Veranstaltung einen Gaming-Event. 
Wenn Sie diese Punkte beachten, wird Ihr Infoabend mit Sicherheit ein Erfolg werden. Denken Sie immer daran: Gaming ist etwas Gutes und Sie möchten damit arbeiten. Seien Sie vorbereitet auf Kritik. Nehmen Sie die Kritik ernst. Hören Sie den Kritikern zu und versuchen Sie, die vorhandenen Befürchtungen zu verstehen. Gerade wenn es um die Sorgen von Eltern geht, ist es wichtig, dass Sie nicht nur zuhören und diskutieren, sondern auch, dass Sie die berechtigten Befürchtungen und Gedanken in Thre Arbeit einfließen lassen. Es geht nicht nur um Gaming, sondern um verantwortungsvollen Umgang damit. Und Ihnen muss klar sein, dass es noch sehr viele Menschen gibt, die die Aufgabe einer Bibliothek darin sehen, Bücher auszuleihen und ein Ort der Ruhe zu sein.

Wenn möglich, spielen Sie die Vorträge vorher durch. Und versuchen Sie, dabei die mögliche Kritik durchzuspielen.

\section{Zusammenfassung:}

Name der Veranstaltung: Infoabend Gaming

Zielsetzung: Das Umfeld der Bibliothek informieren und aktivieren.

Benötigte Ressourcen: Computer inkl. Peripherie, Spiele, Komsolen, Leinwand, Beamer, Raum

Zeitrahmen: 3-4 Stunden (Vorbereitung ca. 3 Tage)

\section{Coding und Modding}

Wir haben nun bereits über die Idee gesprochen, dass die Teilnehmer selbst Games entwickeln. Kudo-Workshops sind ein guter Einstieg in das Thema. Jedoch gibt es noch weitaus mehr Möglichkeiten, die Kreativität der Spieler zu nutzen und zu fördern. Und somit kommen wir zu den Bereichen „Coding“ und „Modding“.

Coding meint die Programmierung von Spielen. Es ist also vergleichbar mit den Kudo-Workshops. Der Unterschied ist der, dass hier wirklich mit Programm-Code gearbeitet wird. Es geht also um Programmiersprachen wie Java, Visual Basic, C++ etc. Es gibt immer mehr Projekte, bei denen die Teilnehmer das Programmieren lernen, indem sie ein Spiel programmieren. Die Fähigkeit, programmieren zu können, ist auch für eine zukünftige berufliche Laufbahn interessant. Der Bedarf an Programmieren ist immer noch hoch. Gaming ist hier ein idealer Einstieg. Natürlich ist die Zielgruppe derer, die sich daran beteiligen wollen, geringer und natürlich ist es zudem weitaus schwieriger, passende „Lehrer“ zu finden. Das alles sollte Sie aber nicht davon abschrecken, nach einem Weg zu suchen, mit dem sich etwas in diesem Bereich machen lässt.

Natürlich kann man auch klassische Programmierkurse anbieten, aber Gaming ist viel cooler. Wenn Sie Coding und Gaming miteinander vernetzen, dann können Sie eine völlig neue Zielgruppe erreichen. Im Laufe des Prozesses werden Games entstehen. Es werden mit Sicherheit einfache Games sein. Das bedeutet, es entstehen keine epochalen Spiele. Aber diese Games sind bei Ihnen in der Bibliothek entstanden und sie gehören von nun an zu Ihrem Bestand. Im Bestand haben bedeutet dabei, dass Sie diese Spiele auch nutzen können. Die Spiele gehören aber nicht Ihnen. Ein gutes Beispiel für ein Projekt in diesem Bereich ist das von AMD initiierte Projekt „Changing the Game“. Hierbei entwickeln die Teilnehmer betreut von Experten aus verschiedenen Bereichen ihr eigenes Serious Game. Ein wie ich finde noch spannenderes Projekt ist „Code-Combat“. Dieses Spiel ist entwickelt worden, um Programmieren zu lernen. 
Man bewegt seine Spielfiguren, aber nicht mit einem Controller, sondern man gibt Befehle in der Programmiersprache Java-Script ein. Man lernt also Programmieren über das Spielen. Dabei ist es den Entwicklern dieses Angebots wichtig zu klären, dass es sich bei CodeCombat nicht um eine Lernsoftware mit Spielelementen handelt, sondern dass es wirklich ein Spiel ist, und man quasi nebenbei Programmieren lernt. Das Modell ist letztlich vergleichbar mit dem Ansatz, den Eli Neiburger auf seiner Vortragsreise in Deutschland beschrieben hat. Er berichtete von seinem Sohn, der durch das Spielen von Pokemon Lesen lernt, da überall im Spiel neue Worte auftauchen, die relevant für das Spielen sind, die der Spieler aber nicht kennt. So muss er seinen Vater bzw. sein Umfeld fragen, was das jeweilige Wort bedeutet, um weiterspielen zu können.

Das Thema Modding (Mod abgekürzt für Modifikation) ist eng verwandt mit Coding. Es geht hierbei um die Veränderung bzw. Weiterentwicklung von schon vorhandenen Spielen. Es gibt eine Vielzahl an Mods und einige davon waren sehr erfolgreich. Es gibt eine große Community an Moddern, die kontinuierlich neue Modds veröffentlichen. Ein gutes Beispiel ist das Spiel „Half Life“ bzw. „Half Life 2“. Zu diesen Spielen gibt es eine Vielzahl an Mods und einige davon sind weltberühmt. Ein bekanntes Beispiel ist sicherlich „Counterstrike“ welches noch heute gespielt wird, und welches zudem immer wieder wegen angeblicher Gewaltdarstellungen bzw. der Motivation, Gewalt auszuüben, kritisiert wurde.

Bibliotheken werden nur in Ausnahmefällen eigene Workshops für Coding und Modding anbieten können. Es werden nur sehr wenige Bibliotheksmitarbeiter in der Lage sein, hier als Lehrer zu arbeiten. Dies ist aber kein Problem. Auch ich habe noch keine Programmiersprache gelernt. Und doch können Bibliotheken mit dem Thema einiges anfangen.

Bibliotheken sollten denjenigen, die sich mit Coding und Modding auskennen, eine Plattform bieten, damit in der Bibliothek kontinuierlich solche Workshops angeboten werden können. Sie werden mit Sicherheit in Ihrem regionalen Umfeld jemanden finden, der an einer Kooperation interessiert ist. Im Vordergrund sollte der Spaß stehen. Es geht eben nicht darum, eine Programmiersprache zu erlernen, sondern darum, ein Spiel zu entwickeln. Am besten wäre es, wenn nicht jeder Teilnehmer sein eigenes Spiel entwickelt, sondern wenn es Gruppen gibt, in denen die Mitglieder gemeinsam an einem Spiel oder einer Mod arbeiten.

Die Bibliothek kann aber noch mehr tun. Sie kann auch hier eine Plattform für die Präsentation der Ergebnisse sein. Spiele werden nicht zum Selbstzweck programmiert. Spiele sollen gespielt werden. Sie können also ein Gesamtkonzept entwickeln, in dem die Entwicklung und die Präsentation der Spiele im Vordergrund stehen. Es müssen keine epochalen Meisterwerke entstehen. Gerade einfache und kurze Spiele werden sehr gerne gespielt, weil man sich danach wieder anderen Dingen z. B. der Arbeit widmen kann. Coding und Modding eignen sich auch hervorragend für Kooperationen mit Unternehmen. Das Interesse an guten Programmierern ist immer noch sehr groß. Gleichzeitig beschweren sich sehr viele Unternehmen über die Tatsache, dass es immer weniger gute Auszubildende gibt. Hier können Sie als Bibliothek konkret helfen. Das Unternehmen stellt Hard- und Software zur Verfügung und die Bibliothek bietet den Raum und die Tatsache, dass sie einer breiten Öffentlichkeit bekannt ist.

Coding und Modding sind mit Sicherheit nichts für Anfänger. Wenn Sie gerade erst anfangen, sich mit dem Thema Gaming über die Grenzen eines Bestandes hinaus zu beschäftigen, ist dies ein Level zu hoch für Sie. Aber Sie können das Wissen um Coding und Modding in Diskussionen nutzen. Denn diese beiden Bereiche zeigen sehr deutlich, warum Gaming mehr ist als ein sinnentleerter Zeitvertreib. Sie können diese Idee auch mit den mit Ihnen verbundenen Schulen besprechen. 


\section{Zusammenfassung:}

Name der Veranstaltung: Coding und Modding

Zielsetzung: Das Umfeld der Bibliothek informieren und aktivieren.

Benötigte Ressourcen: Computer inkl. Peripherie, Spiele, Konsolen, Leinwand, Beamer, Raum

Zeitrahmen: 3-4 Stunden (Vorbereitung ca. 3 Tage)

\section{Gaming-Tage 2.0}

Über Summer Games und Infoabende habe ich schon geschrieben. Nun haben wir natürlich einen sehr wichtigen Punkt noch nicht im Rahmen der Bibliotheksarbeit behandelt: Gaming-Tage. Mit Gaming-Tagen sind Veranstaltungen gemeint, bei denen es nicht mehr alleine um Computerspiele, sondern vielmehr um die ganze Welt des Spielens geht. Es geht also um Brettspiele, Rollenspiele und alle möglichen weiteren Formen und Arten von Spielen. Für Bibliotheken ist es sehr sinnvoll, die einzelnen Spiele-Bereiche miteinander zu vernetzen. Zum einen gibt es dort wirklich eine Verbindung, es sind alles Spiele bzw. spielende Menschen. Zum anderen ist es manchmal notwendig, dem Thema ein bisschen seine exotische Note zu nehmen. Ich weiß, dass Gaming ein sensibles Thema ist. Immerhin geht es dabei um moderne Technologien, um Zeit, um Arbeit, um Virtualität usw. All diese Dinge werden immer wieder kritisch hinterfragt. Das Hinterfragen ist nicht falsch, aber es führt manchmal zu Stillstand. Wenn wir Gaming und Spielen wieder zusammenbringen, dann kann dies sowohl innerhalb der Bibliothek als auch in ihrem Umfeld für ein bisschen Ruhe sorgen.

Natürlich kann man so etwas auch in Vorträgen abhandeln. Man kann erklären, dass Gaming an sich nichts Neues ist, dass es quasi die normale Weiterentwicklung einer der ältesten Kulturtechniken ist. Man kann heute nachweisen, dass Gaming eine Vielzahl an Vorteilen mitsichbringt. Man kann all die vielen Studien zitieren, die letztlich jedem Menschen vor Augen führen müssten, wie wichtig Gaming ist. Man kann diesen theoretischen Weg gehen, aber es ist nicht ansatzweise vergleichbar mit der eigenen Erfahrung, dem eigenen Ausprobieren.

Und dabei geht es auch und vor allem um ein Wiederentdecken der eigenen Spielfreude. Wir leben in einer Welt, in der Spielen bzw. Spielelemente aus dem Alltag nahezu verdrängt worden sind. Dabei gibt es genügend Beispiele dafür, dass Menschen gerade diese Aktivität schätzen. Nehmen wir als Beispiel nur den Karneval. Viele Kritiker des Gamings haben im Laufe der Jahre vergessen, dass sie gerne Spielen. Und viele erwachsene Menschen in meinem Alter haben vergessen, dass sie eine GamingVergangenheit haben.

Wenn Sie einen solchen Tag anbieten möchten, dann sollten Sie am besten einen Parcours entwickeln, den die Besucher durchlaufen können. So können die Besucher den Zusammenhang zwischen Spielen und Gaming besser verstehen. Wenn Sie es möchten, können Sie jeden Teilnehmer danach interviewen.

Ein solcher Tag eignet sich auch, um die Kunden zu bitten, eigene Spiele mitzubringen. Dies betrifft dann vor allem die digitalen Spiele. Das wichtigste sind jedoch Gespräche mit den Kunden über das Thema Spielen und Gaming. Hier können Sie in zwangloser Atmosphäre über alle möglichen Fragestellungen in dem Kontext diskutieren. Wichtig ist, dass für alle Besucher klar wird, dass für Ihre Bibliothek sowohl Spielen als auch Gaming zur Bibliotheksarbeit dazugehören. 


\section{Zusammenfassung:}

Name der Veranstaltung: Gaming-Tage

Zielsetzung: Das Umfeld der Bibliothek informieren und aktivieren.

Benötigte Ressourcen: Computer inkl. Peripherie, analoge und digitale Spiele, Konsolen, Leinwand, Beamer, Raum, Brettspiele etc.

Zeitrahmen: 1 Tag (Vorbereitung ca. 3 Tage)

\section{Reisen zur Gamescom und Co}

Diese Aktivität ist eine der wichtigsten Handlungen überhaupt. Es ist dabei völlig egal, wie groß Ihre Gaming-Aktivitäten werden sollen, ein Besuch der Gamescom sollte für jeden Mitarbeiter einer Bibliothek Pflicht sein. Es gibt also zwei Varianten für diese Aktivität.

1. Besuch der Gamescom und/oder einer anderen Veranstaltung, die sich mit Gaming beschäftigt für die Mitarbeiter der Bibliothek.

2. Besuch der Gamescom mit den Bibliothekskunden.

Beginnen wir mit der Frage, warum Sie unbedingt die Gamescom besuchen müssen. Sie werden vielleicht schon einmal die Frankfurter Buchmesse oder einen Bibliothekartag besucht haben. Diese beiden Veranstaltungen sind bei Bibliotheksmitarbeitern sehr beliebt. Dabei geht es nicht nur darum, Informationen für die tägliche Arbeit zu bekommen. Es geht beispielsweise beim Besuch der Buchmesse auch um die mit den Büchern verbundene Buchkultur. Nun haben einige Bibliotheken Games im Bestand, aber nur wenige Bibliotheksmitarbeiter haben bis jetzt die Gamescom besucht. Dabei gibt es kaum einen anderen Ort, an dem Sie einen großen Teil der Gaming-Kultur so hautnah erleben können wie auf dieser Veranstaltung. Es geht also nicht nur darum, sich zu informieren. Ihre Aufgabe ist vielmehr die Kultur zu verstehen und auf sich wirken zu lassen. Das bedeutet, Sie bekommen hier einen intensiven Kontakt zu Ihren potentiellen Kunden.

Eine große Schwäche von klassischen Konferenzen wie dem Bibliothekartag ist die, dass es sich dabei um geschlossene Systeme handelt. Die Bibliotheksmitarbeiter kommen zusammen, um über die Menschen zu reden, aber nicht mit ihnen. Es gibt keine Bibliothekskunden auf dem Bibliothekartag. Dabei wäre es so wichtig, sie dabei zu haben, damit man die Bibliothek wirklich kundenfreundlich gestalten kann. Sie können noch so viele Statistiken und Analysen lesen. Das direkte Gespräch mit Ihren Kunden ist mindestens genauso wichtig.

Auch beim Dialog mit Gamern geht es nicht nur um das Verstehen der Kundenwünsche - wobei in den meisten Fällen Gamer noch nicht wirklich zu Ihren Kunden gehören - es geht auch darum, dass Gamer verstehen, dass eine Bibliothek ein Gaming-Ort sein kann.

Auf der Gamescom werden Sie eine Vielzahl an Dingen lernen können. Sie werden unglaublich viele Spiele ausprobieren. Sie werden eSports und Shows erleben. Es wird laut und bunt und wild sein. Und dann werden Sie erleben, wie diese Menschen mit Hochtechnologie umgehen. Sie erleben, was man alles aus einem Computer, einem Smartphone oder einem Tablet rausholen kann. Manches wird Sie abschrecken oder amüsieren. Und natürlich werden Sie viele Spiele sehen, die Sie gar nicht in der Bibliothek nutzen können. Am Anfang werden Sie beeindruckt sein. Wenn Sie sich dann an alles gewöhnt haben, werden Sie anfangen zu lernen. Sie werden danach 
Ihre Social Media-Aktivitäten überdenken. Und Sie werden verstehen, warum es nicht ausreicht, eine Konsole aufzustellen, um eine Gaming-Library zu werden.

Aber die Gamescom ist nicht nur für Sie da. Sie können auch Ihre Kunden einladen, mit Ihnen zusammen die Messe zu besuchen. Es ist zwar auch wieder ein Angebot, welches man auch ohne Sie als Bibliothek nutzen kann, aber Sie können es so gestalten, dass es etwas Besonderes wird. So können Sie den Besuch der Gamescom in der Bibliothek vorbereiten. Sie können damit auch vielen Eltern die Sorge nehmen, dass ihr Kind alleine auf der Gamescom ist. Der Eintritt ist übrigens niedrig. Er liegt 2013 zwischen 11,00 € und 15,00 €. Für eine echte Gaming-Bibliothek ist der gemeinsame Besuch der Gamescom ein wichtiger Programmpunkt.

\section{Zusammenfassung:}

Name der Veranstaltung: Besuch der Gamescom

Zielsetzung: Lernen, Informieren und Gamer den Zugang zur Gamescom ermöglichen. Benötigte Ressourcen: Zeit, Eintrittskarte, Geld für Anreise

Zeitrahmen: 1 Tag (Vorbereitung ca. 1 Tag)

\section{Spieletester}

Nachdem Sie nun schon einiges im Bereich Gaming unternommen haben, werden Sie feststellen, dass Ihre Gamer zu Experten geworden sind. Genauer gesagt sind die Gamer schon sehr früh wahre Experten. Sie sollten sie als Experten ernst nehmen und ihnen die Möglichkeit geben, ihr Wissen anzuwenden. Machen Sie die Gamer zu Spieletestern. Die Idee ist ganz einfach. Die Spieletester testen Spiele hinsichtlich vorab definierter Parameter. Natürlich können nur Spiele getestet werden, die auch für die Altersgruppe der Tester zugelassen sind. Ein wichtiger Punkt ist die Auseinandersetzung mit den Spielen. Es geht also nicht nur darum herauszufinden, welche Spiele beliebt sind. Man kann die Parameter so definieren, dass man sich intensiv Gedanken zu bestimmten Fragestellungen macht.

Das Testen von Spielen ist ein elementarer Bestandteil der Gaming-Kultur. Es gibt eine Vielzahl an Magazinen und noch mehr Blogs, in denen Spiele besprochen, analysiert und natürlich bewertet werden. Ich treffe auf der Gamescom immer wieder verschiedene Blogger, die teilweise ein beeindruckendes Detailwissen zu Spielen haben. Nicht ohne Grund achten die meisten Anbieter von Computerspielen darauf, auch die Bloggerszene zu begeistern. Gleiches gilt für die schon beschriebenen Let‘s PlayVideos. Sie müssen also auf jeden Fall den Prozess des Testens sehr ernst nehmen.

Was Sie als Bibliothek dafür brauchen, ist Raum, Zeit, Offenheit und natürlich die dafür notwendige Hard- und Software.

Es gibt verschiedene Varianten für dieses Programm und im Folgenden möchte ich drei Varianten beispielhaft beschreiben:

1. Der Kindersoftwarepreis „Tommi“. Dieser Preis wird vom Family Media Verlag und dem Büro für Kindermedien FEIBEL.DE herausgegeben. Der Preis wird einmal im Jahr verliehen und für Bibliotheken hat er den großen Vorteil, dass das Bewerten der Spiele bereits seit langem in Bibliotheken stattfindet. Es liegen also ausreichend Erfahrungswerte vor, die es Bibliotheken einfacher machen, daran teilzunehmen. Die sog Kinderjury besteht aus mehr als 3.000 Kindern im Alter zwischen 6 und 13 Jahren. Die Spiele werden gespielt und von den Kindern 
bewertet. Die Preisverleihung findet auf der Buchmesse in Frankfurt statt. Als Bibliothek können Sie sich einfach bewerben. Es lohnt sich auf jeden Fall.

2. Natürlich ist der Kindersoftwarepreis nur für eine ganz bestimmte Zielgruppe gedacht. Das Ziel ist der Aufbau von Medienkompetenz und natürlich die Leseförderung. Mit der Altersklasse von 6 bis 13 hat man einige wichtige Zielgruppen nicht erreicht. Hierzu gehören vor allem Gamer im Alter zwischen 13 und 18 Jahren. Nun geht es dabei nicht nur um andere Spiele, sondern auch um andere Fragestellungen bzw. Bewertungskriterien. Deshalb können Sie nicht einfach das Konzept der 6-13-Jährigen in dieser Zielgruppe übernehmen. Es geht um andere Spiele, andere Bewertungskriterien und um andere Gamer. Am besten wäre es, wenn Sie die Bewertungskriterien mit den Testern zusammen festlegen. Ebenso wichtig wäre es, dass Sie auch in dieser Zielgruppe sowohl männliche als auch weibliche Gamer finden und aktivieren könnten. Da es in diesem Fall keinen offiziell übergeordneten Preis gibt, ist der Aufwand, eine gewisse Bekanntheit zu bekommen höher. Als Preis für das Mitmachen könnte man z.B. einen kostenlosen Eintritt für die Gamescom anbieten.

3. Eine Zielgruppe, die eigentlich nie gefragt wird, sind die aktuellen und ehemaligen Gamer über 28 Jahren. Diese Gruppe wird in der Regel nicht mehr als Gamer angesehen. Man glaubt immer noch, Gaming wäre ein Kinder- und Jugendthema. Dabei wäre diese Zielgruppe eine spannende Testgruppe, denn diese Gruppe hat eine Geschichte zu erzählen. Es geht bei dieser Gruppe also nicht um das Erlernen von Fähigkeiten, sondern um Spaß und die Reflexion mit der eigenen individuellen Gaming-Kultur. Diese Zielgruppe benötigt eine eigene Ansprache und zudem ein eigenes Konzept, welches an die Lebens- und Arbeitsrealität dieser Gruppe angepasst worden ist.

Wie gesagt gibt es viele verschiedene Möglichkeiten die Zielgruppen für Spieltester zu definieren. Der Tommi ist mit Sicherheit ein idealer Einstieg und es wäre wünschenswert, wenn alle Bibliotheken in Deutschland mitmachen würden.

Wenn Sie eine Gruppe von Spieletestern haben und zudem auch im Bereich der Gamesentwicklung z.B. im Rahmen von Kudo-Workshops aktiv sind, dann sollten auch diese Spiele durch die Spieletester analysiert werden.

Sie können die verschiedenen Altersgruppen auch mischen. In diesem Fall würden die verschiedenen Altersstufen unterschiedliche Bewertungsparameter bekommen. Darüber hinaus sollten Sie bei der gemischten Variante auch ältere Spiele bewerten lassen, damit nicht nur alle Altersklassen, sondern auch deren Spielkultur in das Ergebnis einfließen kann.

Schließlich müssen Sie noch überlegen, wie Sie die Ergebnisse präsentieren wollen. Ich empfehle auch hier eine Mischung aus offizieller Veranstaltung in der Bibliothek und eine starke Präsenz in den sozialen Medien.

\section{Zusammenfassung:}

Name der Veranstaltung: Spieletester

Zielsetzung: Gamer bewerten Games.

Benötigte Ressourcen: Computer inkl. Peripherie, Spiele, Konsolen, Leinwand, Beamer, Raum

Zeitrahmen: mehrere Tage (Vorbereitung ca. 3 Tage) 


\section{Gaming-PC selbst bauen bzw. Case-Modding}

Bis jetzt haben wir uns über Games, Gaming und Gamification Gedanken gemacht. Dabei haben wir noch nicht überlegt, ob es auch Projekte in Bibliotheken geben könnte, die sich mit der Gaming-Hardware beschäftigen. Dabei gibt es auch hier einige Möglichkeiten und spannende Ansätze. Im Bereich der Konsolen sind die Möglichkeiten begrenzt. Im Bereich der Computer ist aber einiges möglich. Ich möchte gerne zwei Ansätze beschreiben:

1. Wir bauen uns einen eigenen Gaming-PC: In den meisten Fällen kaufen sich Menschen, die einen Computer benötigen, einen kompletten bzw. fertigen Rechner bei irgendeinem Händler. Und in den meisten Fällen reicht dies auch ohne weiteres aus. Man möchte mit dem Computer schreiben, im Internet surfen und vielleicht ein paar Urlaubsfotos bearbeiten. Für solche Aufgaben braucht man keine hochgerüstete Rechenmaschine. Möchte man aber moderne PC-Games spielen, und möchte man diese Spiele dann in hoher Auflösung, mit maximalen Details und vielen grafischen Verbesserungen auf einem 24“" Monitor spielen, dann braucht man weitaus mehr als einen Rechner von der Stange. Ein wirklicher Gaming-Rechner ist Hochtechnologie. Natürlich kann man so einen Rechner auch komplett kaufen, aber am besten ist es, man baut sich den Gaming-PC selbst zusammen. Man bestellt also nur die Einzelteile wie z.B. das Gehäuse, das Netzteil, die Festplatten, das Mainboard, den Prozessor, die Grafikkarte etc. und baut daraus seinen Gaming-PC. Dabei gilt es eine Vielzahl an Punkten zu beachten. Dazu gehört die ausreichende Stromversorgung, denn solche Systeme brauchen weitaus mehr Energie. Dazu gehört auch die richtige Kühlung, denn ansonsten geht Ihr Rechner sehr schnell kaputt. Alle diese Punkte müssen bereits bei der Auswahl der Einzelteile beachtet werden. Wenn man den PC zusammengebaut hat, muss man nun das Betriebssystem und alle Treiber installieren, um dann schließlich alles zu testen. Das mag für viele Menschen sehr kompliziert klingen, für jemanden, der dies schon einmal gemacht hat, ist es kein großes Thema mehr. Jedoch ist es selbst für den erfahrensten Gamer immer wieder eine Herausforderung, ein System zusammenzustellen und dann zusammenzubauen.

2. Case-Modding: Nun müssen Sie nicht gleich einen komplett neuen PC bauen. Es kann auch sein, dass Sie mit der Leistung Ihres Systems völlig zufrieden sind. Dann gibt aber immer noch die Option, das Aussehen des Computers zu verändern. Und damit ist nicht gemeint, dass Sie einen netten Aufkleber auf das Gehäuse kleben. Case-Modding ist eine eigene Disziplin und in der professionellen Form eine Kunstform, die mich immer wieder zutiefst beeindruckt. Auf der Gamescom kann man beobachten, wie Case-Modder neue Kunstwerke erschaffen, die „nebenbei“ auch Computer sind.

Auch wenn es sich hier um Phantasiegebilde handelt, sollen die Computer auch funktionieren. Man muss also trotzdem auf die Belüftung, die Energieversorgung etc. achten. Wenn man sich die Ergebnisse ansieht, so erkennt man, dass diese Werke mehr sind als nur Computer. Es sind Skulpturen, Kunstwerke, Denkmäler und sie sind ein elementarer Bestandteil der Gaming-Kultur. Bei Case-Modding geht es nicht um Funktionalität, sondern eher um Originalität und doch würde ich mir einige der Systeme gerne in mein Büro stellen, zeigen sie doch, wie viel Kreativität in der Gaming-Community vorhanden ist. Gamer sind Macher. Sie sind Schöpfer, Künstler, Innovatoren. Und Case-Modding ist ein weiteres Mosaiksteinchen in diesem Bild. 
Für Bibliotheken ergeben sich aus beiden Beispielen erneut Möglichkeiten für Aktionen und Projekte. So können und sollten Bibliotheken einmal im Jahr der Ort für das Bauen eines PCs und/oder für Case-Modding sein. Sie müssen dafür nicht selber einen PC bauen können. Es ist nur wichtig, dass Sie den Raum zur Verfügung stellen und zudem Menschen suchen, die hier helfen bzw. beaufsichtigen möchten. Auf jeden Fall sollten Bibliotheken Ausstellungsraum für die Werke von Case-Moddern sein. Zudem können Sie auf diesem Weg zu einem eigenen professionellen GamingPC kommen. Sie könnten ein Team aufbauen, welches die Aufgabe hat, den ultimativen Bibliotheks-Gaming-PC zu bauen. Natürlich brauchen Sie ein Budget für die Hard- und Software. Aber wenn Sie potentiellen Geldgebern vermitteln, welch vielfältige Kompetenzen Ihr Projekt fördert, werden Sie mit Sicherheit Sponsoren finden. Für die Hardware müssen Sie zum jetzigen Zeitpunkt mindestens 500,- $€$ einplanen. Dafür kriegen Sie dann schon einen wirklich guten Rechner und können die meisten Spiele in maximaler Auflösung und maximalen Details spielen. Hinzu kommen noch Kosten für das Betriebssystem und natürlich die Spiele. Sie benötigen je nach Team zwei bis drei Tage für den Bau eines PCs und/oder ein Case-Modding-Projekt. Natürlich können Sie das alles auch viel schneller bekommen. Ich empfehle aber, daraus einen richtigen Event zu machen. So wäre es toll, wenn die Teilnehmer ihre Arbeit via Blog, Facebook, Youtube etc. live kommunizieren.

Auch bei diesem Projekt können Sie einen Wettbewerb integrieren. Sie starten mit zwei Gruppen und die Gruppe, die zuerst fertig ist, bekommt einen Preis. Sie sollten unbedingt darauf achten, dass Sie auch weibliche Teilnehmer bekommen.

\section{Zusammenfassung:}

Name der Veranstaltung: Case-Modding und Gaming-PC selbst bauen

Zielsetzung: Gamer bauen ihren eigenen Gaming-PC bzw. entwickeln sein Aussehen weiter.

Benötigte Ressourcen: Computer inkl. Peripherie, Raum, Werkzeug, Software, Zugang zum Internet

Zeitrahmen: 1-3 Tage (Vorbereitung ca. 3 Tage)

\section{Gaming-Public-Viewing}

Eine weitere Möglichkeit, die Bibliothek als Gaming-Ort zu etablieren, ist Public Viewing. Wie bereits erwähnt ist eSports ein sehr erfolgreicher Bereich der Gaming-Kultur. Die internationalen Sieger sind wahre Superstars. Und manch ein Event zieht tausende Zuschauer an. Die großen Wettbewerbe werden global durch Pay-TV-Kanäle und teilweise auch kostenlos übertragen.

In vielen Ländern wurden und werden spezielle Bars eröffnet, bei denen es vergleichbar mit den Sports-Bars im Bereich Fussball - darum geht, die jeweiligen Wettbewerbe live anzusehen.

Eine Bibliothek kann ebenfalls ein Ort sein, an dem man Live-Übertragungen aus dem Bereich eSports zusammen erleben kann. Diese Übertragungen kann man auch mit normalen Gaming-Events kombinieren. Alles was Sie benötigen ist Raum, einen Beamer, eine Leinwand und einen Computer, um das gestreamte Signal empfangen zu können. Wenn möglich brauchen Sie noch einfache PC-Lautsprecher, um den Ton ebenfalls übertragen zu können. 


\section{Zusammenfassung:}

Name der Veranstaltung: Gaming-Public-Viewing Zielsetzung: Gemeinsam eSports-Events anschauen.

Benötigte Ressourcen: Computer inkl. Peripherie, Raum, Beamer, Leinwand, Internetanschluss, Lautsprecher

(0) Zeitrahmen: 6 Stunden (Vorbereitung ca. 1 Tag) 